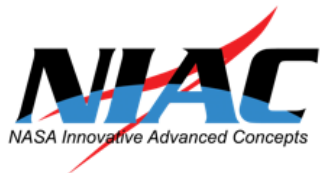

\title{
SPARROW: Steam Propelled Autonomous Retrieval Robot for Ocean Worlds
}

Dr. Gareth Meirion-Griffith ${ }^{1}$, Dr. Daniel Levine ${ }^{1}$, Dr. Timothee Pourpoint ${ }^{2}$, Dr. Kris Zacny ${ }^{3}$, Dr. Cynthia Phillips ${ }^{1}$

${ }^{1}$ NASA Jet Propulsion Laboratory, California Institute of Technology, Pasadena, California

${ }^{2}$ Purdue University, West Lafayette, Indiana

${ }^{3}$ Honeybee Robotics, Pasadena, California

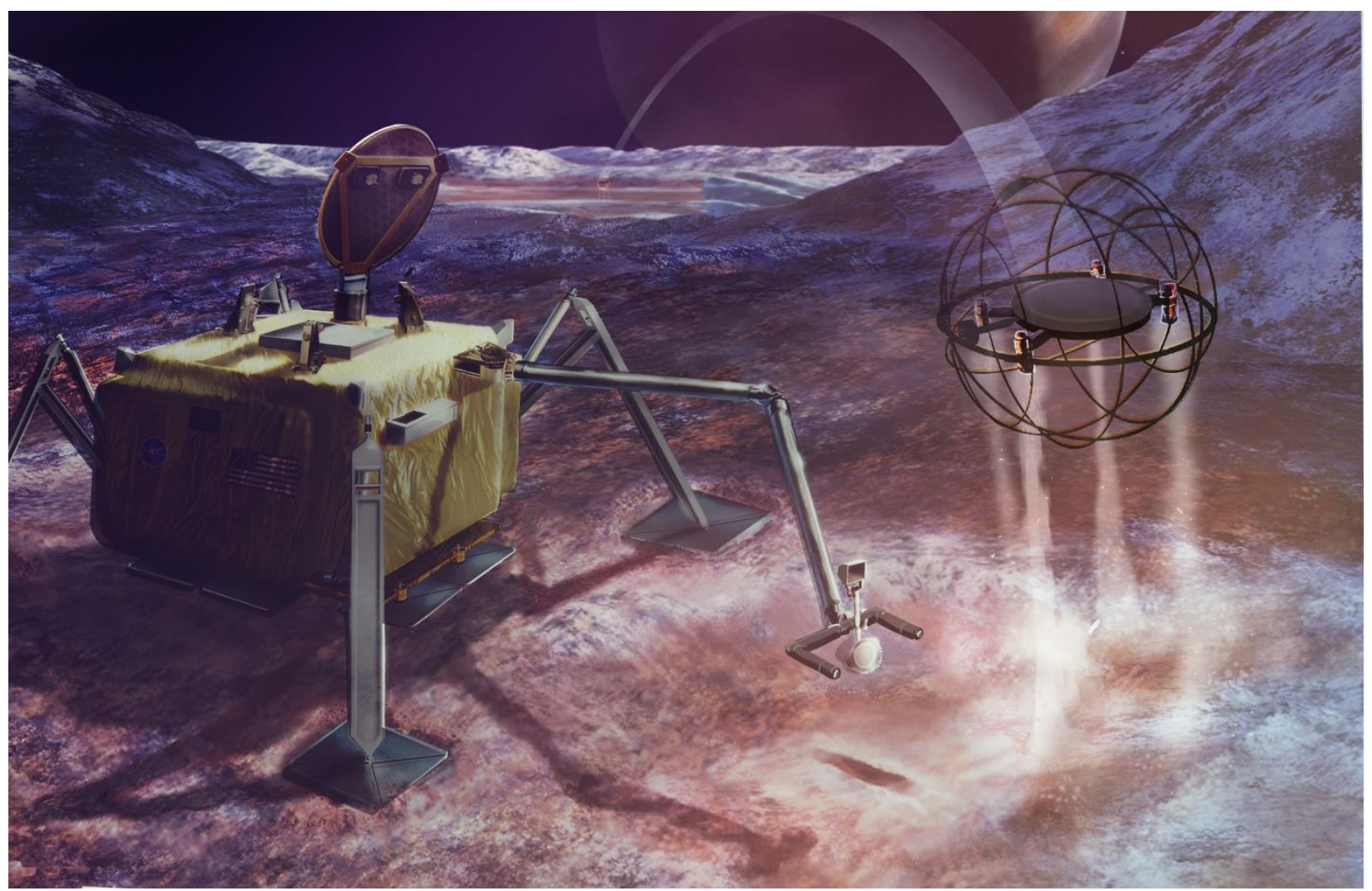




\section{Acknowledgements}

The authors wish to thank the NASA Advanced Innovative Concepts program for support and guidance during the Steam Propelled Autonomous Retrieval Robot study. We also wish to recognize Ben Hockman (JPL), Michael Orth (Purdue University) and Steven Ford (Honeybee Robotics) for their efforts in bringing the study to fruition.

This report contains preliminary findings for discussion purposes only. The content is subject to revision as analysis proceeds. 


\section{Contents}

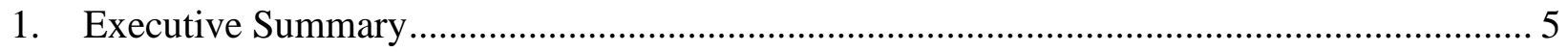

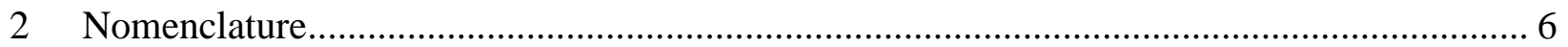

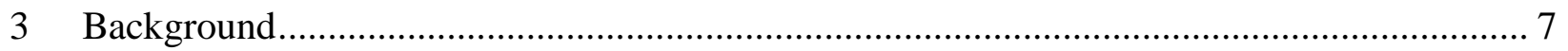

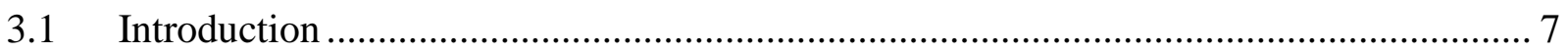

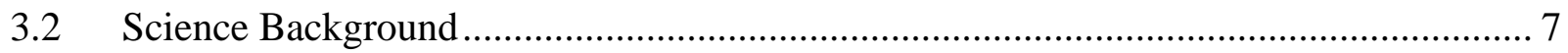

3.3 Science Return as a Function of Mobility ............................................................... 8

1) Local mobility $(0-1 \mathrm{~km})$ within one geologic unit. ………........................................... 8

2) Medium-range mobility $(1-10 \mathrm{~km})$ enabling a partial transect of one geologic unit. ........... 8

3) Long-range mobility $(\leq 100 \mathrm{~km})$ enabling measurements to be made across multiple

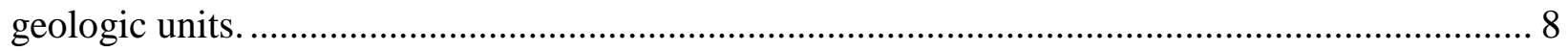

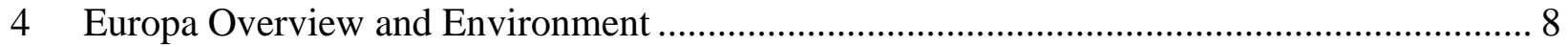

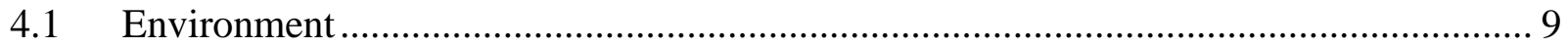

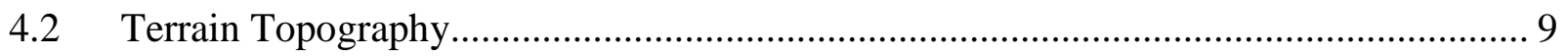

4.3 Knowledge of Surface Mechanical Properties .......................................................... 10

4.4 Some Geotechnical Properties of Unconsolidated Cryogenic Ice ................................ 10

$5 \quad$ Study Approach and Preliminary Mission Architecture..................................................... 11

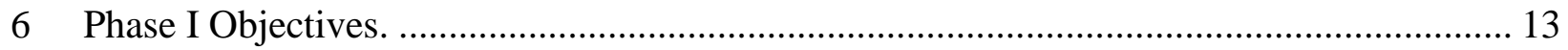

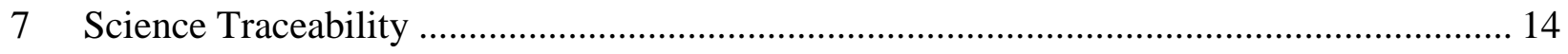

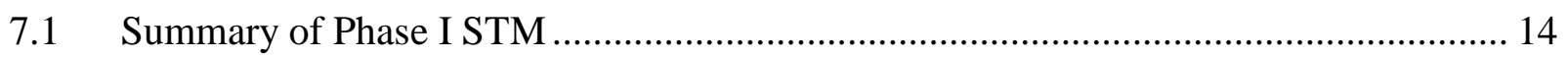

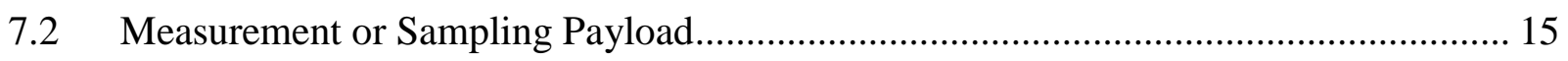

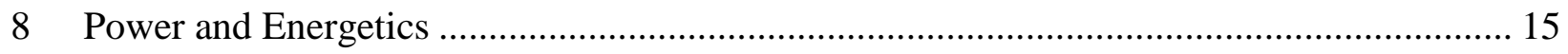

8.1 Propellant Harvesting ................................................................................................ 17

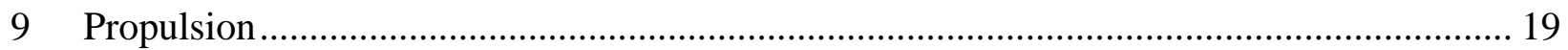

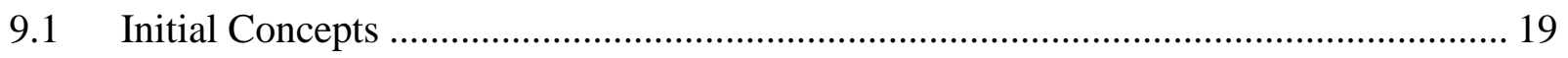

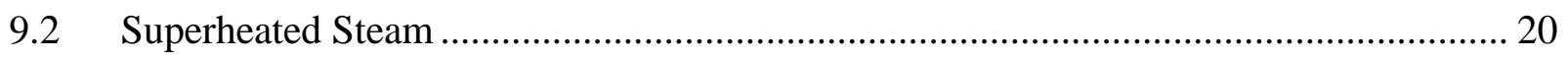

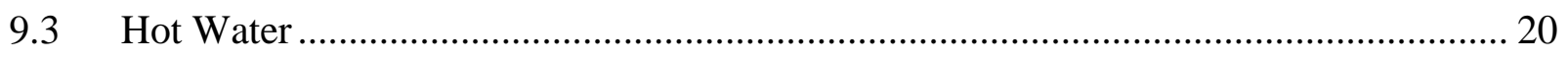

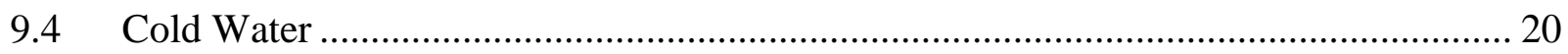

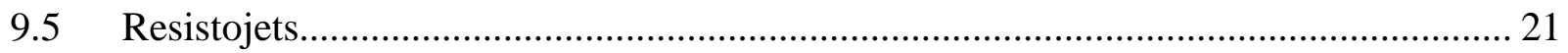

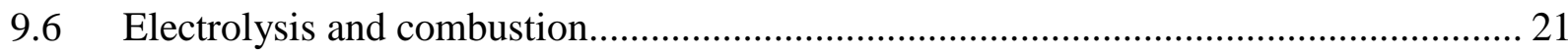

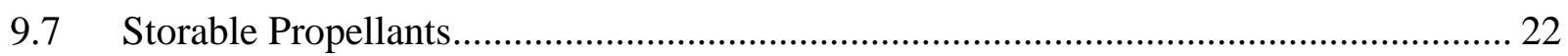

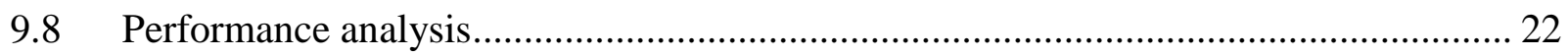

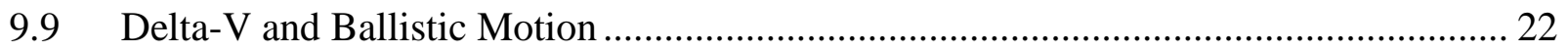

NIAC Grant 17-NIAC18B-0097_ March 2019 


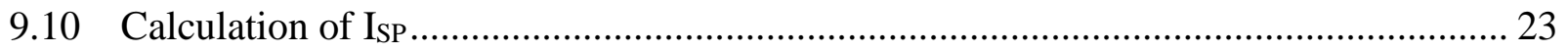

9.11 Results of First-Order Calculations.......................................................................... 23

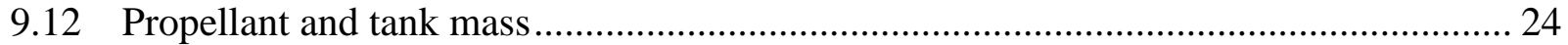

9.13 Propellant Production Energy Requirements ...................................................... 25

9.14 Efficiency Losses due to Cage Drag .................................................................... 26

9.15 Propulsion Strategy Selection and Furthered Analyses .......................................... 28

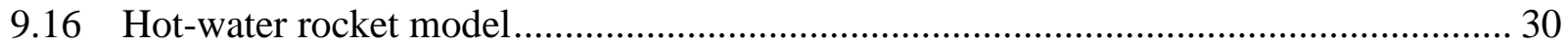

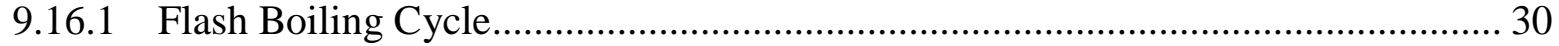

9.16.2 Thermodynamic cycle of a hot water rocket............................................... 31

9.17 Hot Water Rocket Testing ........................................................................... 33

9.18 Effects of Ice Impurities on Propulsive Efficiency ................................................. 36

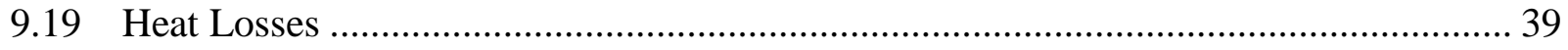

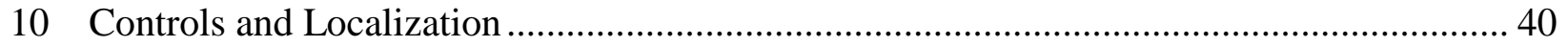

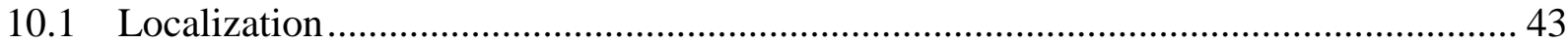

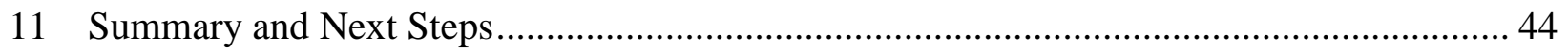

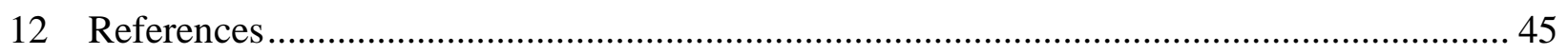

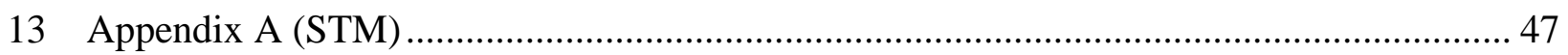




\section{SPARROW: Steam Propelled Autonomous Retrieval Robot for Ocean Worlds}

Gareth Meirion-Griffith, Daniel Levine, Timothee Pourpoint, Kris Zacny, Cynthia Phillips

Jet Propulsion Laboratory

Pasadena, CA, 91109

\section{Executive Summary}

The Steam Propelled Autonomous Retrieval Robot (SPARROW) for Ocean Worlds was a Phase I mission concept study funded under the NASA NIAC program. This report represents the findings of that study and recommendations for future work. SPARROW, envisioned as a soccer ball-sized payload to a primary lander mission, is a propulsively hopping robot for the exploration of Europa's rugged, icy surface. A multi-thruster, passively gimballed robot within a protective, spherical shell, SPARROW is able to freely rotate, self-right, and tumble over chaotic terrains. Europa's abundant surface ice would be harvested as an in situ propellant source. The principal objective of SPARROW is to increase the science return of a Europa landed asset by enabling access to distal, spatially distributed geologic units.

The design of mobility systems for Europa is challenging, due in part to its almost entirely unconstrained surface topography and strength. Images returned by Voyager and Galileo yielded resolutions on the order of hundreds of meters per pixel, with localized regions reaching 6 meters per pixel-still far larger than a typical rover. A key benefit of SPARROW's hopping, impacttolerant design, is that it eliminates the need for a priori information regarding terrain topography and surface strength; no surface reaction forces are required for motion. In this context, SPARROW is believed to be entirely terrain agnostic. In this report we detail the results of three study objectives: i) to quantify the energy required to collect surface ice, change its phase, and maintain propellant temperature, ii) to identify control and estimation strategies that enable SPARROW to successfully reach, and return from, regions of scientific interest, and iii) to characterize the impact of SPARROW's range on likely science return. Five water-based propellant architectures are presented alongside their mass, power, and volume requirements. Monte Carlo simulations of SPARROW hopping and tumbling over $1 \mathrm{~km}$ of glacial ice are summarized, characterizing SPARROW's sensitivity to uncertainty in: initial pose, thrust profile, and vehicle-terrain interaction. A science traceability matrix is presented, which details the effect of sortie range on three science goals: constraining Europa's evolutionary morphology, assessing sub-surface ocean habitability, and searching for life and/or biosignatures. 


\section{Nomenclature}

$I_{S P}:$ Specific Impulse

$\Delta V$ : Delta- $\mathrm{V}$, change in velocity

$V$ : Velocity

$D$ : Distance

$g$ : acceleration due to gravity

$\theta$ : launch angle

$C^{*}$ : Characteristic velocity

$C_{f}$. Thrust coefficient

MMH: Monomethyl Hydrazine $\left(\mathrm{N}_{2} \mathrm{H}_{3}\left(\mathrm{CH}_{3}\right)\right)$

NTO: Nitrogen Tetroxide $\left(\mathrm{N}_{2} \mathrm{O}_{4}\right)$

$O / F$ : oxidizer to fuel ratio by mass

$T_{m i n}$ : minimum propellant tank wall thickness

$P$ : pressure

$r$ : Radius

$\sigma_{\text {yield: }}$ material yield stress

$T_{\text {net: }}$ net thrust

T: Thrust

$D$ : Drag

$\sigma:$ Solidity

$\rho$ : fluid density

$S$ : Surface area

$C_{D}$ : drag coefficient

$\dot{M}$ : Mass flow rate

$V_{e}$ : exit velocity

$A_{e}$ : exit area 


\section{Background}

\subsection{Introduction}

The Steam Propelled Autonomous Retrieval Robot for Ocean Worlds (SPARROW), shown in Figure 1, was a collaborative, NIAC Phase I study, between NASA's Jet Propulsion Laboratory, Purdue University and Honeybee Robotics. Envisioned as a soccer ball-sized payload to a primary lander mission, SPARROW is a propulsively hopping robot, enabling rapid access to spatially distributed regions of scientific interest.

The principal advantage of SPARROW is its terrain and orientation agnosticism; its design and operation requires no a priori knowledge of terrain topography or strength. In this report the concept, mission architecture, and fundamental feasibility of SPARROW are addressed. Considerations for propulsion, energetics, controls, localization, and science operations are described.

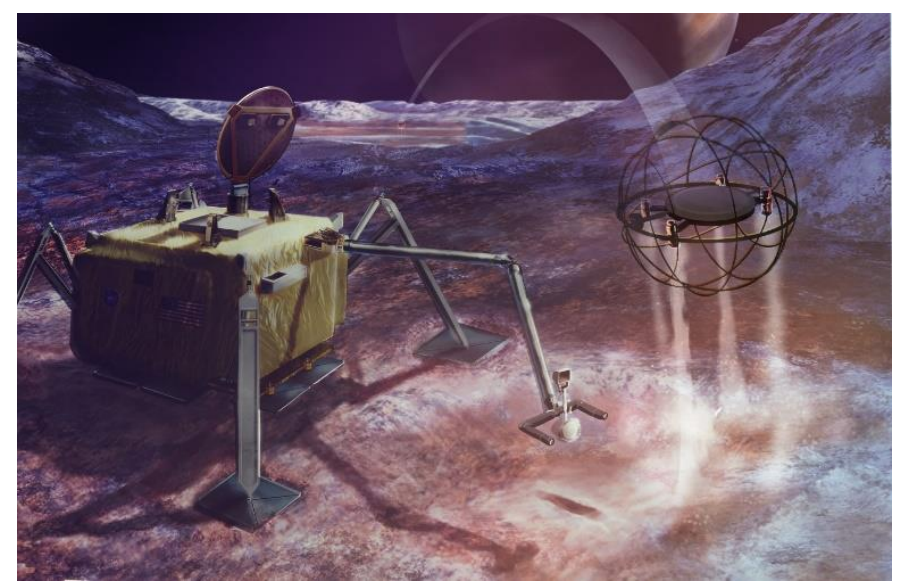

Figure 1: Artists illustration of the SPARROW concept. Figure adapted from (Hand 2016)

\subsection{Science Background}

Images of Europa's surface from the Galileo spacecraft reveal multiple feature types and geologic units, each with a distinct morphology and discoloration thought to be related both to composition and formation mechanism (Carlson, et al. 2009). These features provide clues as to how material is transported from Europa's subsurface ocean to the surface. Identification of mineral species and biomarkers in these regions is likely to hold significant implications for Europa's biological potential, history, and subsurface habitability, as referenced in the Decadal Survey (Squyres 2011). Multiple models exist for the formation of various geologic units on Europa's surface, and as yet there is no scientific consensus on which feature types are the most compelling for future exploration. Scientific considerations for landing site selection include the inferred composition and abundance of non-ice materials, the potential for surface-subsurface exchange, and the desire to land at a relatively young, unprocessed surface location. Landing site selection is further complicated by the fundamental tradeoff between the safety of the landed asset and the potential for enabling discovery. Accordingly, selection of a safe landing site for an immobile asset may be balanced against the capabilities of a mobile payload to reach distal regions of greater scientific interest.

It is the scientific premise of the SPARROW concept that the enablement of multi-site, multigeologic unit exploration holds the potential to greatly increase the science return while reducing the risks associated with landing at a less compelling site. 


\subsection{Science Return as a Function of Mobility}

The exploration of solar system bodies, such as Earth's moon and Mars, has often followed the model of high-coverage remote sensing missions preceding closer inspection via surface in situ operations, first with static landers and successively with mobile assets. NASA has historically staged missions within this model to mitigate the complexity of designing spacecraft in the face of uncertainty surrounding the target operating environment; data collected in early missions can be used to impact the design of higher complexity downstream missions that return to the target body. In recent years, Ocean Worlds have garnered substantial interest from the scientific community, precipitating the Europa Clipper mission and Europa Lander study. If NASA's exploration of Ocean Worlds proceeds in family with the lunar and Martian exploration paradigms, a subsequent surface mobility mission to Europa is a possibility. However, the travel time to Europa is significantly longer than to Mars, and between the present day and the expected arrival of Clipper (mid to late 2020s), further measurements of Europa's surface will not become available. As such, mission concepts that are inherently robust to terrain uncertainties are advantageous.

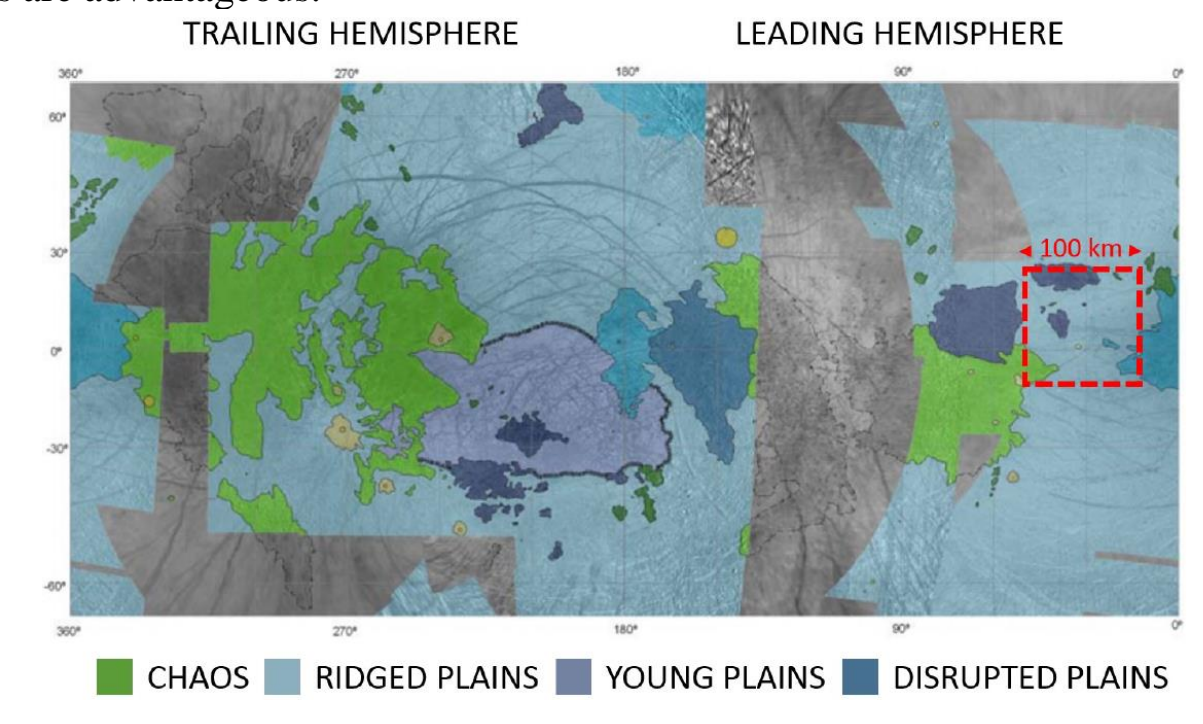

Figure 2: Distribution of geologic units across Europa's surface. Adapted from (Doggett 2007).

Mobility offers many advantages to Europan exploration, including the ability to access topographically extreme regions and to enable science outside of the potentially exhaust contaminated landing zone. Figure 2, adapted from (Doggett 2007), reveals the heterogeneous distribution of geologic units across Europa's surface. Chaos, ridges, bands, and heavily disrupted plains are among its features. Three mobility length scales are emergent:

1) Local mobility $(0-1 \mathrm{~km})$ within one geologic unit.

2) Medium-range mobility $(1-10 \mathrm{~km})$ enabling a partial transect of one geologic unit.

3) Long-range mobility ( $\leq 100 \mathrm{~km})$ enabling measurements to be made across multiple geologic units.

\section{Europa Overview and Environment}

Europa presents challenges to surface mobility that can be broadly grouped into two categories: environment and terrain. 


\subsection{Environment}

Owing to its position within the Jovian radiation environment, Europa's surface is exposed to significant ionizing radiation, largely in the form of electrons (Paranicas, Cooper and Garrett 2009). Within its expected 20 day surface mission, the proposed Europa Lander is anticipated to be exposed to a total ionizing dose of approximately 1.5 Mrad (Hand 2016). This is far in excess of that experienced by previous and current Martian robotic explorers. While some of this dose may be mitigated through the use of shielding and hardening, Europa's radiation environment remains extreme. In the context of a relatively short mission (weeks) and the spacing of scientifically compelling sites, the requirement for rapid mobility becomes evident.

\subsection{Terrain Topography}

Rapid surface mobility on other worlds is challenging for several reasons, including the need for robustness to terrain uncertainty, the necessity of (perhaps fully) autonomous operations, and mitigation of multiple sources of risk. Uncertainty surrounding Europan terrain topography can readily lead to complex robot-ground interactions that are difficult to predict. Images of Europa taken by Voyager and Galileo have resolutions on the order of hundreds of meters per pixel, with localized regions reaching 6 meters per pixel. Such resolution does not provide sufficient knowledge of terrain features at the lander/robot scale to inform designs of terrain-dependent mobility systems.

Ice morphologies such as penitentes (Hobley, et al. 2018), regolith (unconsolidated material) (Buratti and Golombeck 1988), salt evaporites (McCord, et al. 1998), solid ice, and chaos (Collins and Nimmo 2009) have been postulated.
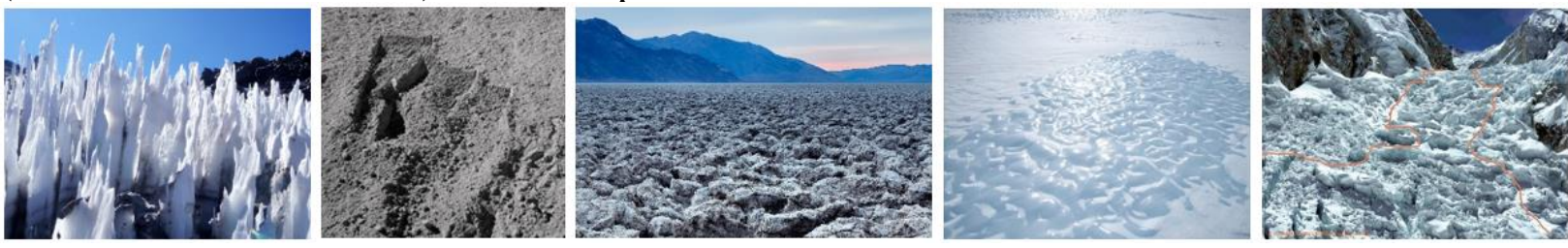

Figure 3: Possible Europa-like terrains. From left to right-penitentes, regolith, salt evaporites, solid ice, chaos.

Depending on the modality with which a robotic system is designed to traverse the Europan terrain, each morphology represents a unique set of challenges. Penitentes, which are likely only possible within $\pm 30^{\circ}$ of the equator, may not be traversable by a ground-based system, depending on their size and spacing. If not sufficiently small or distributed, line of sight would readily become an impediment to path planning, autonomous or otherwise.

As has been experienced on Mars, regolith presents both wheel slip and embedding challenges to their safe operation. Both the Mars Exploration Rovers (MER) and Mars Science Laboratory (MSL) rovers have experienced concerning levels of slip of sandy terrains (Purgatory, Hidden Valley).

Salt evaporates are believed to be formed on Europa when the sublimation of upwelled, brackish material, leaves non-uniform crystalline structures at the surface (McCord, et al. 1998). Similar morphologies are present in some of Earth's dry lake beds such as the region known as Devil's Golf Course, located in Death Valley California. Ground mobility on salt evaporates is yet to be tested, however, its highly rough and undulatory nature is likely to pose both wear and entrapment challenges.

A solid ice surface with few or no regions of unconsolidated material may represent possibly the most challenging scenario for a sampling system; the opposite is true for mobility. If the ice is heavily disrupted, however, as in the case of Europa's well documented chaos terrains, ground-based travel may become extremely challenging. 
While little is known about the surface strength of Europa, the proceeding subsection discusses a possible range based on remote observations and experiments performed in a cryogenic vacuum chamber.

\subsection{Knowledge of Surface Mechanical Properties}

There currently exist no means of remotely determining Europa's surface strength, which dictates both the traction of a wheel (or foot) and the extent to which it will sink into a regolith a critical and often limiting factor in the efficacy of mobility systems. Surface strength is a function of both the material and the weathering processes to which it is exposed.

As the Jovian satellites are airless bodies of a similar age to Earth's moon ( 4.5 b.y.), it may be reasonable to expect that their surfaces would be similar. The regolith found on the surface of Earth's moon is up to $20 \mathrm{~m}$ deep with a mean particle size range of $40-800$ microns (Heiken, Vaniman and French 1991). However, the processes governing the generation of Europa's surface properties are entirely different. Of prime importance is the fact that while Earth's moon is geologically dead, many ocean worlds, including Europa, remain geologically active. Thus, although Europa is 4.5 b.y. old, its surfaces is on the order of $50 \mathrm{~m}$.y. old due to frequent geologic resurfacing (Moore, et al. 2009). This young surface (in geological timescales) has evidence of only very few impact cratering events. Other Jovian and Saturnian satellites such as Callisto, which are geologically near-inactive, bear a markedly more similar surface appearance to Earth's moon, but are not the subject of this report. Given that the particle sizes found on Earth's moon are largely dependent on the age of the surface (Heiken, Vaniman and French 1991), we cannot infer the particle sizes present on ocean worlds from our knowledge of our moon.

On Europa, surface ice is subject to multiple regolith-producing forces: tectonics, micrometeoroid bombardment, charged particle impacts, and the possibility of plume ejecta deposition (Moore, et al. 2009). Although past measurements have penetrated only the remote sensing layer (up to $\sim 10$ wavelengths), they are in agreement with a highly porous, unconsolidated surface. Photometric and thermal inertia measurements made by the Galileo spacecraft indicate void fractions (free space to grains) on the leading and trailing sides on the order of 0.25 and 0.79 , respectively (Buratti and Golombeck 1988). Regolith grain sizes in the range of 20 to several hundreds of microns have been reported (Hansen 2005), and the surface thermal inertia is 20 times lower than the value expected for solid water ice (Morrison 1977).

\subsection{Some Geotechnical Properties of Unconsolidated Cryogenic Ice}

In 2016, under NASA STMD prime contract NNN12AA01C, geotechnical tests were performed by $\mathrm{G}$. Meirion-Griffith to ascertain a range of shear and bearing strengths of unconsolidated, particulate ice. Tests were performed inside a cryogenic vacuum chamber at

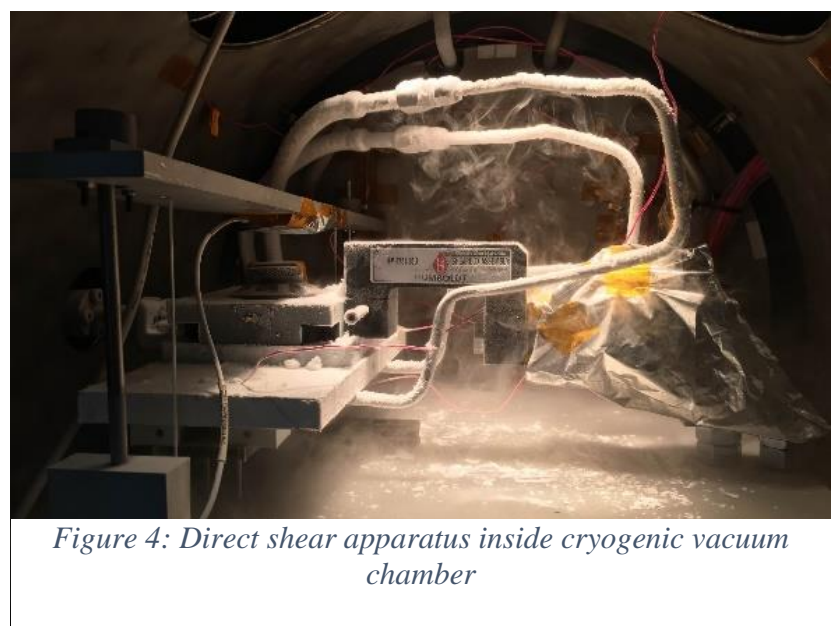


near vacuum $\left(\sim \times 10^{-5}\right.$ Torr $)$ and temperatures ranging from $-130^{\circ} \mathrm{C}$ to $-195^{\circ} \mathrm{C}$. Ice temperature was monitored using thermocouples. The temperature at which each ice specimen stabilized was a function of its mass: smaller test specimens tended towards $-130^{\circ} \mathrm{C}$ due to radiative heat loss. Two types of tests were performed: direct shear, Figure 4, and bearing capacity. The purpose of the tests was to identify a range of strength parameters than can be used in terramechanics models to predict vehicle performance. Tests were performed on both spherical and angular ice grains with diameters ranging between $10 \mu \mathrm{m}$ and $2 \mathrm{~mm}$.

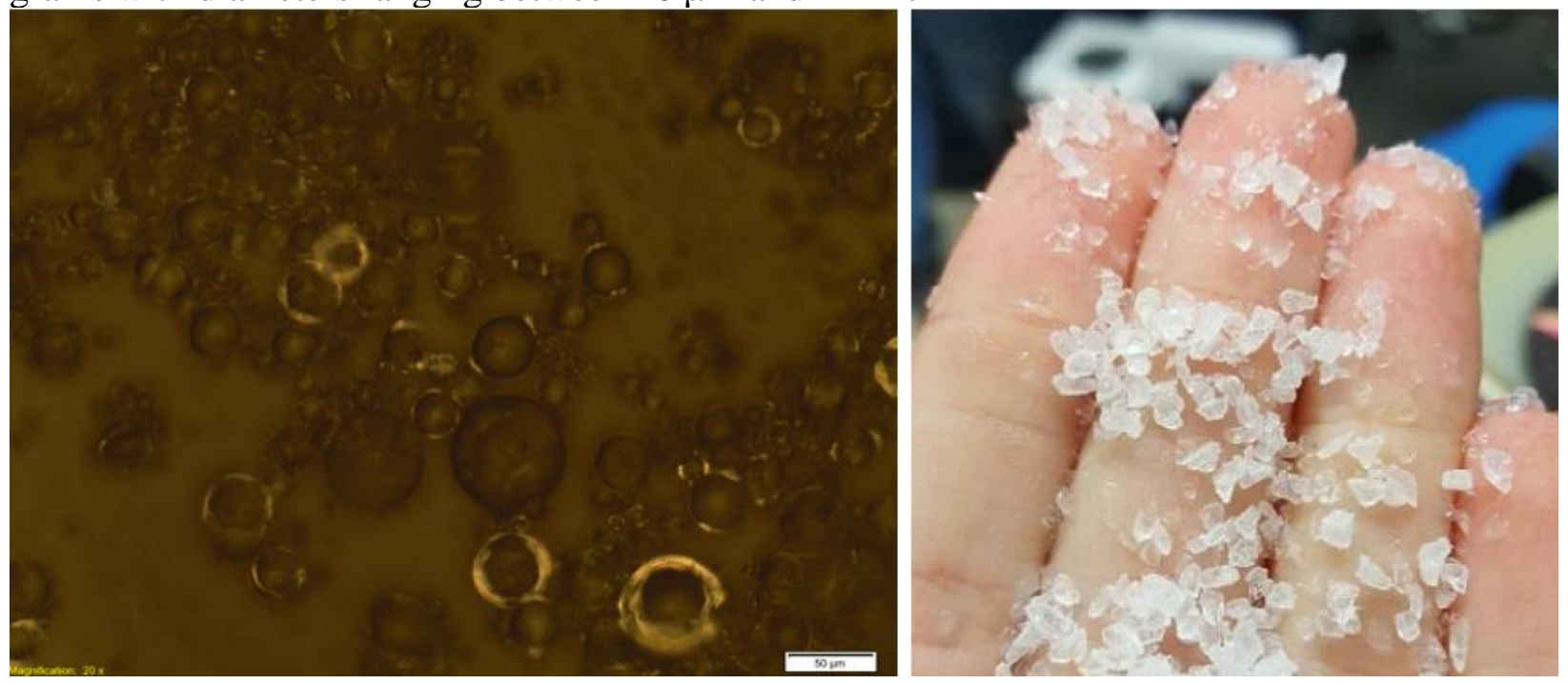

Figure 5: Spherical atomized ice grains (left) and angular $2 \mathrm{~mm}$ ice grains (right)

The results of these tests showed that under Europan conditions, unconsolidated ice regoliths may hold strengths ranging from that of gravel to a material significantly weaker than snow on Earth. Such uncertainty is further exacerbated by the presently unknown timescales of ice sintering on Europa. Ice sintering is an under-researched area, but has garnered recent interest from Molaro (Molaro, et al. 2019 (In press)). The true strength of this surface and its topography will not be known prior to the first landing.

\section{Study Approach and Preliminary Mission Architecture}

In the face of significant uncertainty surrounding terrain properties, it is prudent to ask how one can reasonably defend the efficacy of a proposed mobility system design. Myriad mobility concepts exist as variations of wheeled, tracked, and legged systems. All, however, rely on a priori knowledge of the surface they are intended to traverse. A promising alternative to more traditional ground-based mobility architectures is that of a hopper. Hoppers holds the distinct advantage of minimizing time spent in contact with the terrain. However, conventional mechanical hoppers rely on a relatively strong terrain on which to impart reactive forces and are, thus, terrain-dependent. In the possible case of a weak, loose regolith, such mechanisms may be inefficient or fail. Inspired by the (Kalantari and Spenko 2014) and GimBall (Figure 3) (Floreano and Wood 2015) robots, SPARROW features a central module passively gimballed inside a protective, spherical shell. Contrary to the use case for HyTAQ and GimBall, Europa's lack of an atmosphere precludes the use of rotor flight. Rather, SPARROW replaces the quadrotors with a thruster configuration, enabling it to operate in a vacuum environment. A key innovation of the SPARROW concept is the use of a propellant harvested from an abundantly available in situ material: water ice. Water-based propellants have previously been used for for low-thrust, onorbit attitude control (James, et al. 2017) (Rowen, et al. 2018). 


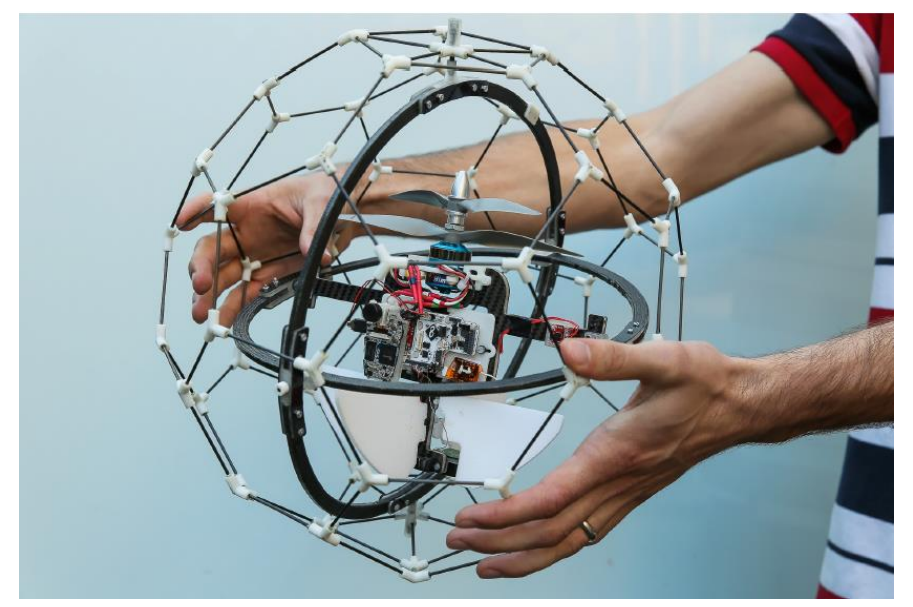

Figure 6: The Gimball robot [FW15].

With the primary lander as SPARROW's base, ice (propellant) extraction from Europa's surface would be performed through the incorporation of Honeybee Robotics' Europa Drum Sampler into the lander arm. Akin to SPARROW's terrain agnosticism, EDuS' ice harvesting capabilities (but not efficiency) are independent of surface roughness. EDuS' rotating cutter head throws shavings behind a buffer plate, which when inverted, deposits the ice into the heating chamber through a funnel. Figure 7 depicts the key components of EDuS' design.

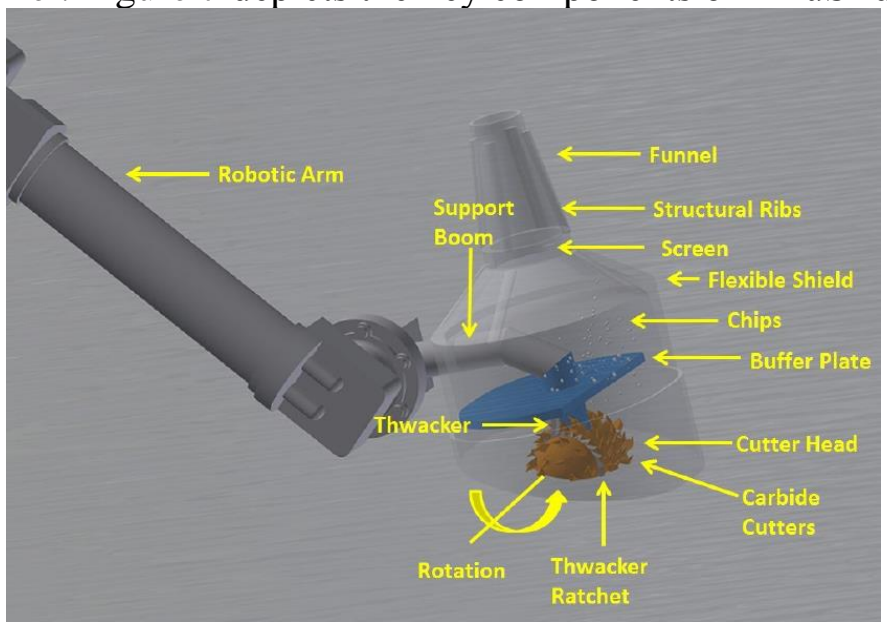

Figure 7: The Europa Drum Sampler (EDuS) major components.

As illustrated in Figure 8 and expanded upon here, there are several phases of SPARROW's operation:

1. The lander arm, equipped with the EDuS ice collector, is deployed to the surface. The requisite mass of ice is acquired and transferred to SPARROW.

2. Using the $250 \mathrm{~W}$ of waste-heat generated by a general purpose heat source (GPHS) internal to the propellant tank, the ice undergoes a phase change and is heated to several hundred Kelvin.

3. SPARROW is released by the lander arm and pulses its thrusters to roll away from the lander, clearing any potential collision zone.

4. Once clear, SPARROW orients itself in the direction of its first target and briefly exhausts steam through its nozzles, placing it on a ballistic arc to the target. 
5. SPARROW performs a brief retro-thrust above the surface prior to an uncontrolled landing and subsequent tumble toward the remote geologic target. Being able to freely rotate within its protective cage, SPARROW would come to rest at an orientation amenable to measurement activities.

6. With the measurement complete, SPARROW would use its remaining propellant to hop back to the lander, making a controlled landing at a safe standoff. Pulsing its thrusters, SPARROW maneuvers itself within the lander arm's reach for re-capture, charging, and measurement data transfer. Note that the propellant required for the return hop will always be less than that for the outbound hop due to the reduction of the wet mass.

SPARROW's terrain-agnosticism and rapid-traverse capabilities enable the development of a mobility platform capable of achieving compelling scientific objectives within Jupiter's lifespanlimiting radiation environment. By housing its payload and avionics inside a passively-gimballed cage, SPARROW is capable of $360^{\circ}$ thrust vectoring, can safely tumble into complex topographies, and self-right prior to measurements. The innovative use of an ISRU harvested propellant is well aligned with NASA Technology Roadmap areas 2.1.6 (warm gas) and 2.1.7 (micro-propulsion) and has several intrinsic benefits:

1. For a mission concept with the explicit intent of detecting native surface chemistry, a reduction in the deposition of foreign materials on the surface significantly alleviates many contamination and planetary protection concerns.

2. It places no additional mission requirements to carry consumables.

3. It enables multiple re-charge and hop sorties within a short mission.

4. It presents an opportunity to store water in such a way as to offer increased radiation shielding.

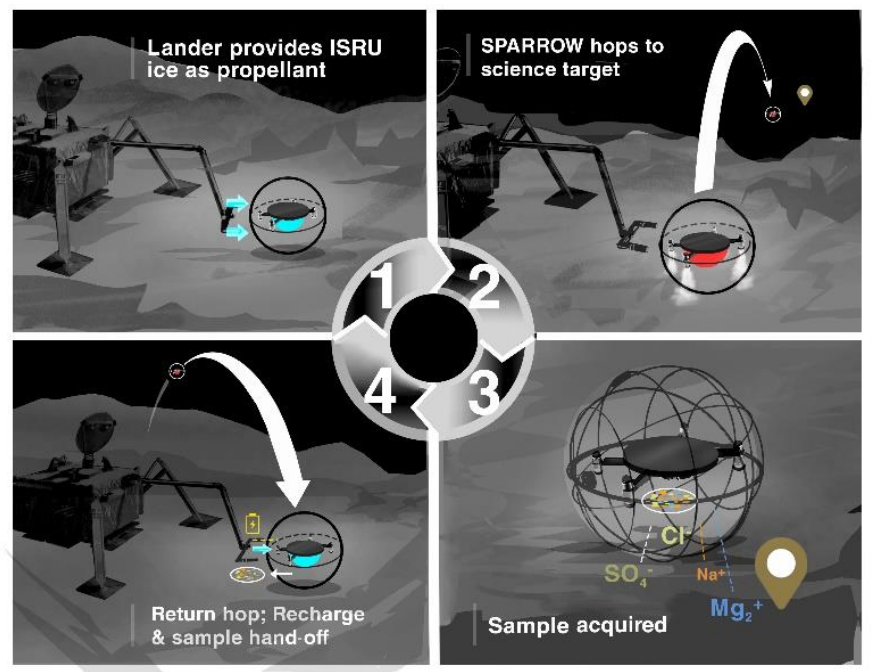

Figure 8: The SPARROW concept of operation.

\section{Phase I Objectives.}

This Phase I study focused on three facets of the mission architecture representing the most pressing feasibility concerns:

1. Energetics: Quantify the energy required to collect surface ice, change its phase, and maintain propellant temperature 
2. Controllability and Localization: Identify control and estimation strategies that enable SPARROW to successfully reach, and return from, regions of scientific interest

3. Science Operations: Characterize the impact of science payload capabilities on system sizing transfer. As with many NASA missions, science return is the primary motivation for the SPARROW concept. Likely science return as a function of SPARROW's capabilities is used to drive system-level requirements and addressed in the following section.

\section{Science Traceability}

This section details the results of a science traceability matrix (STM) developed under this task. The STM illuminates the relationship between the potential for discovery and multi-site exploration, providing context from which engineering requirements can be derived. Here we present a distillation of the STM, which shares the goals and objectives of the 2016 Europa Lander Study report (Hand 2016) and is augmented to highlight the effect of mobility.

\subsection{Summary of Phase I STM}

At the highest level, the STM's three goals are:

1. SG1: Biosignatures: To search for evidence of life, past or present, on Europa.

2. SG2: Habitability: To assess the habitability of Europa via in situ techniques.

3. SG3: Geophysics: To characterize surface morphological and geophysical properties.

There is no single, conclusive test for the presence of life (Goal SG1). However, when considered as a biosignature-collective, organic, inorganic, and morphological indicators may be used to suggest the presence of life. Biosignatures include, but are not limited to: patterns among molecules (e.g., carboxylic and amino acids), cell-like structures, surface discoloration, and biominerals such as $\mathrm{S}_{\mathrm{i}} \mathrm{O}_{2}$. Spatial variation in the detected number and type of biosignatures is highly likely and multi-site measurements increase both the probability of biosignature detection and our understanding of their spatial diversity.

Whether or not Europa reveals evidence of life, the assessment of regional habitability (Goal SG2) and the relationship between surface and subsurface ocean (Goal SG3) are important. These goals may be assessed by studying the composition of non-ice species and measuring their proximity to liquid water and/or recently-erupted material.

The Phase I STM used three example length scales as the basis of discussion for likely, mobility-enabled science return: $1 \mathrm{~km}, 10 \mathrm{~km}$, and $100 \mathrm{~km}$. All length scales hold common advantages over a static asset in that they greatly increase the measurement area, reduce the risk of landing in a scientifically less compelling region, and enable measurements outside of the anticipated lander-exhaust contaminated zone. Beyond this, SPARROW's ability to partially or fully interrogate geologic units grows monotonically with range. Within $1 \mathrm{~km}$ of the lander, SPARROW would enable mobility within a single geologic unit, enabling lander-proximal sorties into heavily disrupted material and increasing the likelihood of encountering unit contacts. A $10 \mathrm{~km}$ sortie would enable a transect across a smaller geologic unit or the partial interrogation of up to 3 units. Long-range mobility (up to $100 \mathrm{~km}$ ) would enable complete transects of one or more geologic units. For the purpose of this study, particular importance was emplaced on partial or full transects; while terrain closer to the interior of a geologic unit is likely younger and may represent freshly upwelled material, the outer reaches are likely to have 
been formed at the feature's inception. In this scenario, a partial or full transect would interrogate the historical record of the feature, subsuming both age and possible means of formation.

Moreover, mobility across multiple geologic units enables selection between multiple existing habitability hypotheses through comparisons of their historical records and interrogation of the relationships between them. In this manner, the ability to reach multiple units holds the significant advantage of providing relational insights over a broader range of Europa's surface features. The full STM is provided in Appendix A of this report.

\subsection{Measurement or Sampling Payload}

Maintaining SPARROW's low-mass, volume, and cost objectives all but precludes the integration of a robotic arm, sampling mechanism, or on-board sample processing. Sampling in particular adds significant cost to any surface mission. The selection of a threshold science payload, defined as the minimum set of instruments capable of satisfying the STM's goals, was thus performed as a measurement-only study. The proposed instrument package is that of a context imager (Goal 3) and imaging spectrometer (composition, Goals $1 \& 2$ ). Note that while a seismic package is likely to be included in the design of a static lander, one has not been selected for SPARROW; it is believed that mobility within $100 \mathrm{~km}$ of the landing site is unlikely to unlock significant, additional seismic information. Moreover, a seismic package would likely require SPARROW to remain in place for one tidal cycle, approximately 3.5 Earth days, reducing its ability to reach multiple targets in rapid succession.

Context imagers, such as the Mars Science Laboratory's (MSL) navigation and mast cameras (NCAM/MCAM), provide ground-truth, which may be used to refine data/images obtained by more remote assets such as Europa Clipper. They may also provide information regarding the provenance of deposited material and the detection of plume vents not visible from the static lander. Spectral imagers, such as the Compact Reconnaissance Imaging Spectrometer for Mars (CRISM) or MSL's MastCam (MCAM) would enable the detection of various minerals via the use of lens filters. This may result, as has been the case for CRISM, in the selection of scientifically compelling regions for future operational planning. Microscopic imagers, such as MSL's Mars Hand Lens Imager (MAHLI) would enable the detection of small-scale surface properties. On Mars, such instruments are used to examine the micro-structure of rocks and regolith, which in the context of Europa would be used to refine our knowledge of surface mechanical properties. Microscopic imagers may also be able to observe cell-like structures, if present.

While context imagers are high-TRL, feasibility concerns over SPARROW's ability to perform meaningful compositional analyses on Europa persist. It has been widely reported, and best summarized by (Nordheim, Hand and Paranicas 2018) that Europa's surface is highly irradiated, placing impetus for measurements to be made beneath the immediate surface, preferably at depths exceeding $10 \mathrm{~cm}$. The development of subsurface measurement strategies is a critical and open question requiring further investigation. Investigation into the enablement of subsurface measurements has been proposed for Phase II.

\section{Power and Energetics}

SPARROW's most critical feasibility concern is energetics. The collection of surface cryogenic ice, transformation into propellant, and thermal maintenance are energetically expensive. The principal energy requirement for the SPARROW mission concept stems from the manufacturing of a viable propellant from ISRU cryogenic ice. This requires a $\Delta \mathrm{T}$ of several hundred Kelvin (Europa's surface temperature seldom exceeds $100 \mathrm{~K}$ (Ashkenazy 2016)) and one or two phase changes depending on the selection of propulsion architecture. Three sources of energy were considered: batteries, radioisotopes, and solar. Of the three, solar is the most readily rejected. Figure 9 shows the results of a simulation conducted to compare the incident light 
available at the surfaces of Europa and an Alaskan Glacier in early summer. Note that the timescale for Europa is ten years, while a representative sample of only four days was used on Earth to clearly demark the variation as a function of time of day. It is clear that Europa's peak solar irradiance is less than an order of magnitude lower than that available on Earth. Such reduced irradiance would yield the requirement for a massive and voluminous solar array and is not considered a credible option.

A battery only option for a Europan asset, as discussed in the 2016 Europa Lander Study Report (Hand 2016), represents an attractive solution due to its relatively low cost and architectural simplicity. However, assuming an energy density of up to $260 \mathrm{Wh} / \mathrm{kg}$ (representative high-performance LIB) and 100\% thermal efficiency, a $5 \mathrm{~kg}$ SPARROW would require a $3 \mathrm{~kg}$ battery to perform a single $1 \mathrm{~km}$ round-trip sortie. As the required mass of propellant grows proportionally with range, so too does the required battery mass. Continuing the example of a single $1 \mathrm{~km}$ round-trip sortie, the required battery mass to heat sufficient propellant for a $10 \mathrm{~kg}$ dry-mass SPARROW consumes almost the entire system mass.
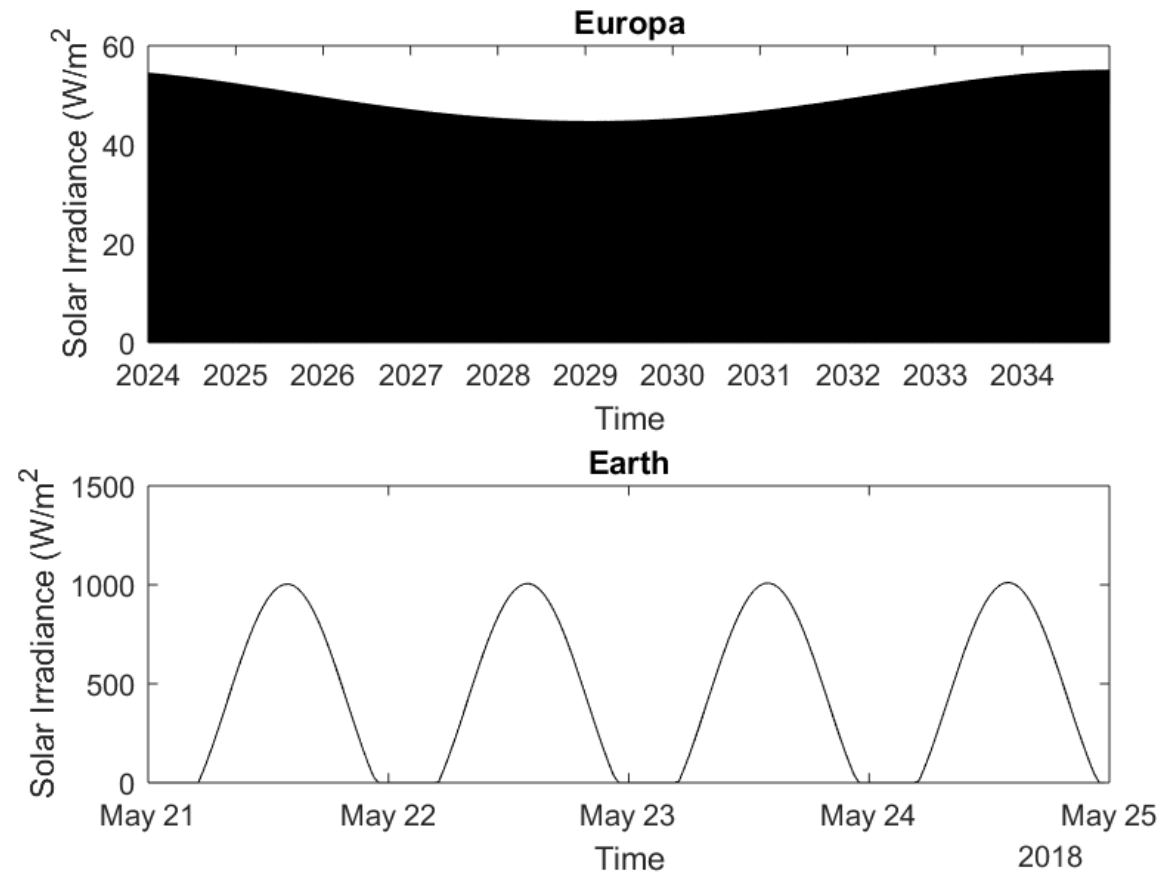

Figure 9: Solar irradiance comparison between Earth and the Europan equator

Radioisotope thermoelectric generators (RTG), as utilized on multiple NASA missions, have the distinct advantage of almost limitless energy, albeit at relatively low power. While the multimission RTG (MMRTG) is too large of a subsystem to fit within SPARROW's payload-sized concept, its core component, the general purpose heat source (GPHS), provides an attractive option. Although a single GPHS provides only approximately $5 \mathrm{~W}$ of electrical power, it can provide $250 \mathrm{~W}$ of continuous thermal power at a volume of only $5 \times 10^{-4} \mathrm{~m}^{3}$ and a mass of $1.5 \mathrm{~kg}$ (Dudzinski 2009). Assuming negligible radiative or conductive losses, such a GPHS would be capable of heating the ice required by a $5 \mathrm{~kg}$ SPARROW to hop $1 \mathrm{~km}$ in only 2.5 hours. A $20 \mathrm{~kg}$ (dry mass) SPARROW would require 52 hours of heating to enable a $10 \mathrm{~km}$ round trip sortie with a propellant mass of $16 \mathrm{~kg}$. 


\subsection{Propellant Harvesting}

The efficiency of ice excavation using Honeybee Robotics' (Co-I Zacny) Europa Drum Sampler (EDuS) concept was evaluated as a function of weight on bit (WOB), ice temperature, and salinity. An experimental, rapidprototyping approach was adopted over theoretical or computational methods; the fracturing mechanics of cryogenic ice is a sufficiently burgeoning area of research as to warrant early proof-ofconcept. Initial tests were aimed at evaluating the general viability of EDuS. A sample
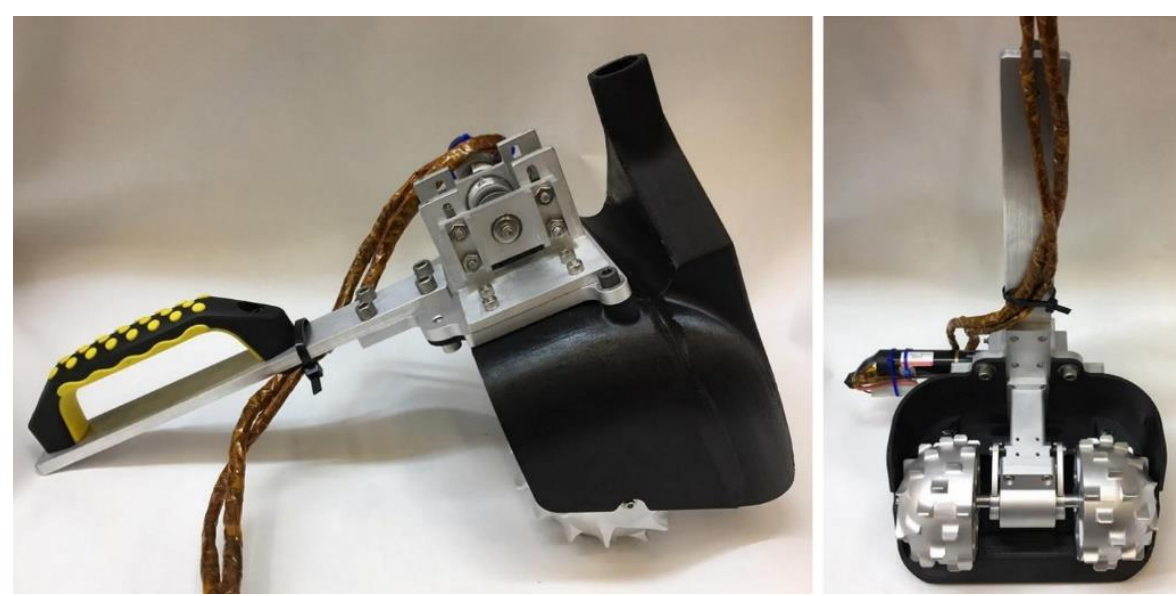

Figure 10: Manually operated EDuS prototype collection shell was attached to the front to demonstrate the collection and transfer of sample, and a handle was attached to allow the user to manipulate the instrument as a robot arm might, as shown in Figure 10 . The $48 \mathrm{~V}$ motor was commanded to spin in velocity control mode at $25,000 \mathrm{rpm}$, which after the gearhead, corresponds to a cutter speed of $1316 \mathrm{rpm}$. Tested in pure water ice, it was demonstrated that the cutter heads could shave ice from the surface when pressed down. Too much weight on bit, however, caused the motor to stall, tripping the motor controller's 10A current limit; while a larger motor, gearhead, or a more capable controller would improve this, further iterations of the EDuS prototype were required to maintain an average power consumption in the 50-200W range.

Figure 11 shows the second EDuS prototype, consisting of a dual cutter-head, passive $Z$-stage with mass-hanger, DC brushless motor, collection buffer, and associated electronics. Tests were performed on both pure and Epsom salt-saturated ice at $-20^{\circ} \mathrm{C}$ and $-86^{\circ} \mathrm{C}$ in three preparations: loose, compacted "snow", and solid. While the variables and temperature range addressed in Phase I experiments were not exhaustive, several key trends were observed. Collection efficiency increased nearlinearly as a function of WOB and temperature. Efficiency degraded with increased ice salinity and compaction. The least efficient test (solid ice, $86^{\circ} \mathrm{C}$, salt-saturated) recorded ice excavation occurring at 400 $\mathrm{kJ} / \mathrm{kg}$, significantly lower than the Phase I proposal estimate of $700 \mathrm{~kJ} / \mathrm{kg}$. This number may be

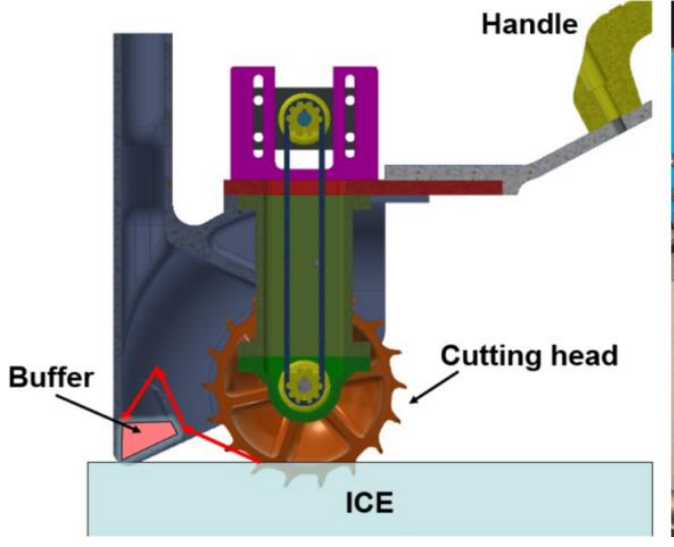

Figure 11: EDuS prototype developed and tested under Phase I

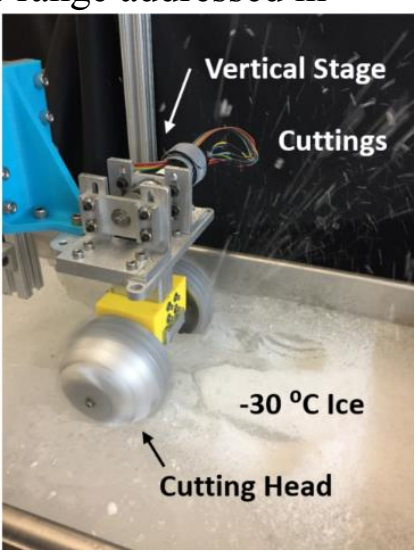

approached, however, at surface temperatures of $\sim 100 \mathrm{~K}$. Figures 12, 13, and 14 show EDuS' measured performance as functions of power consumption vs WOB, excavation rate vs WOB, and excavation efficiency. 


\section{Electrical Power Draw vs WOB}

- Power Data - - Linear (Power Data)

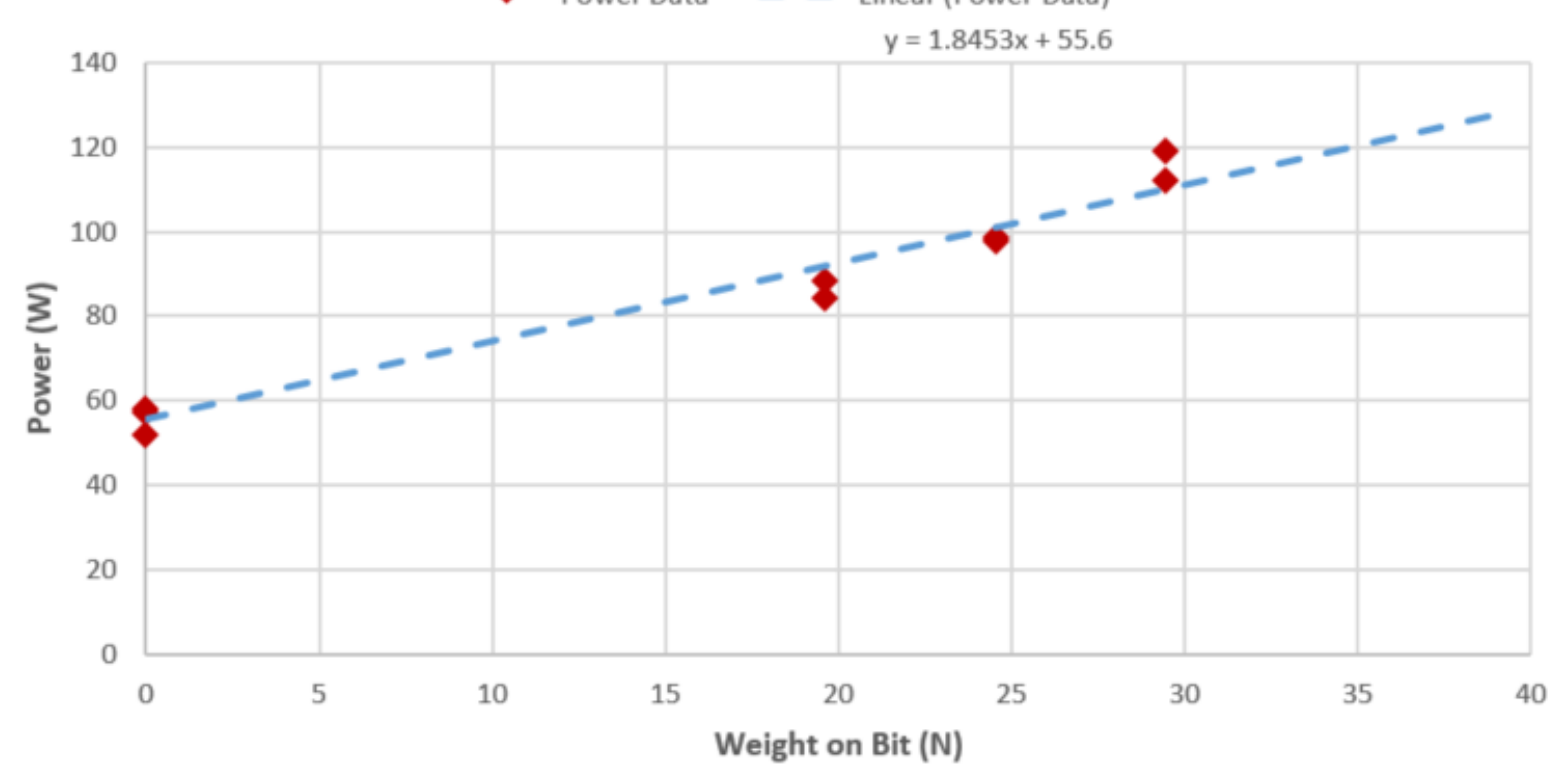

Figure 112: EDuS' power consumption as a function of WOB

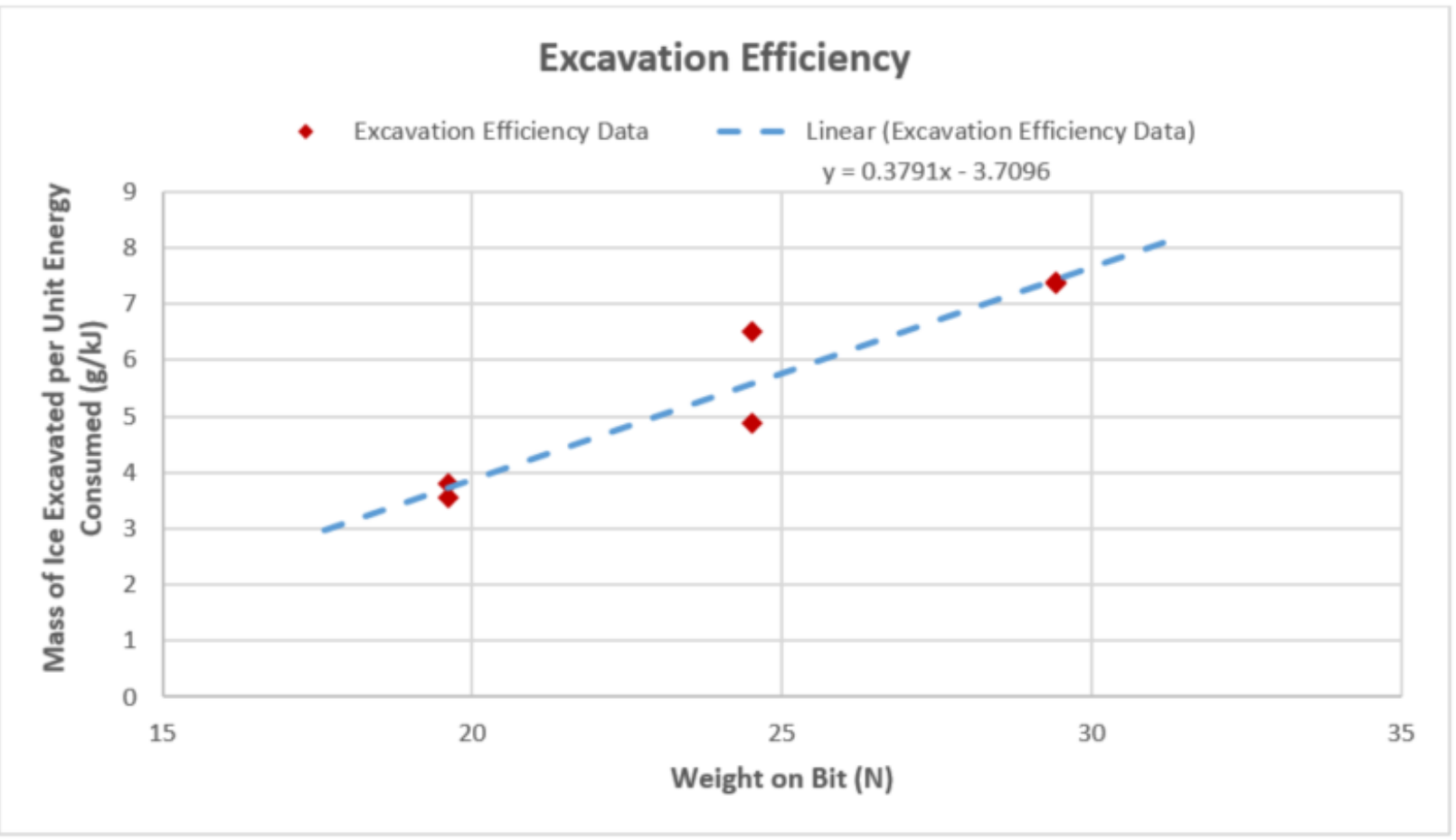

Figure 13: EDuS' excavation rate per $k J$ 


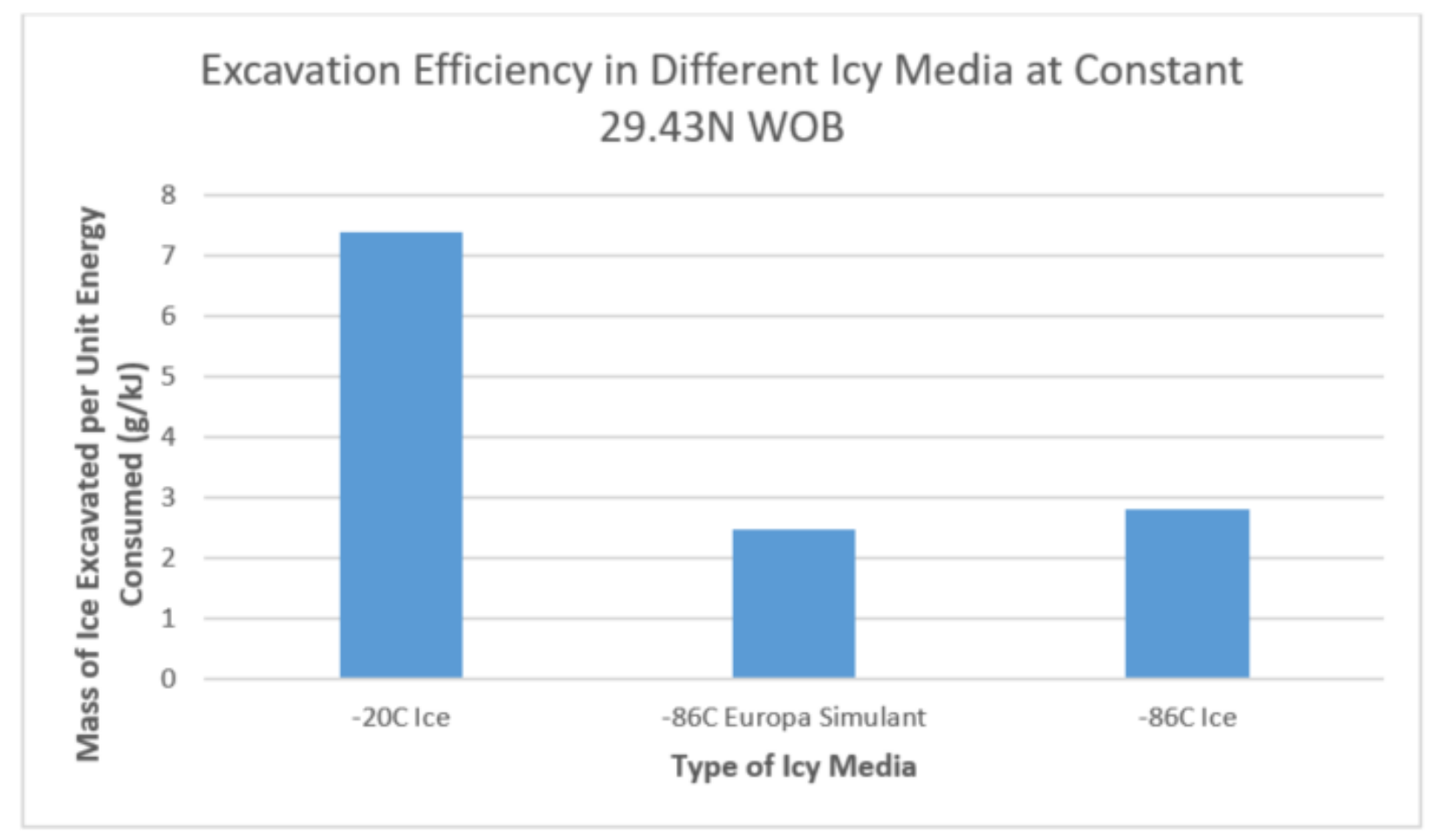

Figure 14: Relative efficiencies of EDuS on various ices

\section{Propulsion}

In this Section the results of analyses into five water-based propulsion architectures and their perceived potential for enabling discovery under the SPARROW concept are discussed. Any propulsion system is defined by its power to weight ratio. SPARROW's propulsion system is no different, however, its unique operating environment and architecture may be used to define key metrics required to select an appropriate strategy. While SPARROW's thrust-level requirements are relatively benign thanks to Europa's vacuous and low-gravity environment, the minimum impulse bit requirements for highly controlled and three-dimensional maneuvers are challenging. Additionally, the use of cryogenic ice, eventually transformed into a motive gas emplaces engineering challenges unique to SPARROW.

In order to achieve sufficient mobility per the STM, sortie ranges on the order of $5 \mathrm{~km}$ from the lander, to be repeated multiple times, are desired. For the purpose of propulsion strategy selection, any system incapable of providing $5 \mathrm{~km}$ of travel (10 km roundtrip) was deemed unsatisfactory. Further, the size of the SPARROW vehicle and its support infrastructure should not exceed that of a secondary payload to a primary mission. It is conceivable that the result of the SPARROW study may be to indicate that SPARROW is capable of flying only as a prime mission. This, however, was not the objective of the Phase I study.

\subsection{Initial Concepts and General Description}

The Phase I study considered six options for SPARROW's propulsion needs; five using ISRU water-ice and one requiring the use of storable propellants from Earth. The study of a storable propellant, MMH-NTO, was performed as the basis of comparison for SPARROW's more uncommon architectures. The five steam-based concepts are: 1) superheated steam, 2) hot water, 3) cold water, 4) a resistojet, and 5) an electrolysis-produced liquid bi-propellant. These are discussed in the following subsections. It should be noted that the choice of Hastelloy propellant tanks is ubiquitous across all propulsion systems presented here; Hastelloy has excellent thermal 
properties and is resistant to corrosion both by salts and sulfuric acid, both of which are believed to be pervasive across Europa's surface.

\subsection{Superheated Steam}

Superheated steam represents the simplest of the propulsion concepts considered in Phase I, both conceptually and mechanically. The steam, heated to approximately $1250 \mathrm{~K}$ would be stored in gas phase and exhausted through an inert-gas, blow-down thruster. Figure 15 shows a sketch of a pure steam thruster's configuration. Steam thrusters can achieve specific impulses on the order of 100 to 150 seconds, depending on the temperature to which the steam is stored. As a blow-down thruster, however, both thrust and specific impulse experience nonlinear reductions during firing; tank pressure and temperature cannot be maintained. As shown in Table 2 and Table 3, the low storagedensity of steam, even at high pressures, results in the need for a large and consequently massive tank.

\subsection{Hot Water}

During operation of a hot-water thruster, ice/water is heated to its saturation temperature and pressure just below the critical point. One advantage of the hot water configuration over the steam option outlined in the previous section is its higher storage density since the water is still in a liquid state within the tank. Upon expulsion through the nozzle the liquid experiences rapid volumetric expansion, causing it to flash-boil. The resulting

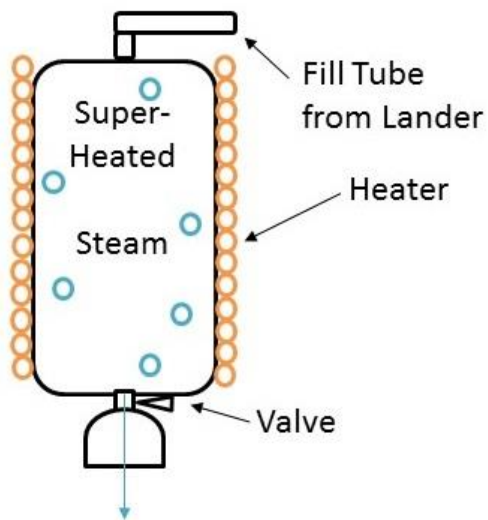

Figure 15: Sketch of superheated steam rocket concept steam produces thrust. A sketch of the concept is shown in Figure 16. A review of water-based thruster research found related experimental results reported by Kolditz et al. (M. P. Kolditz 2004). These results suggest an advantage over pure blowdown thrusters due to the attenuation of feed pressure drop; during firing, the partially-evacuated ullage causes any remaining liquid to evaporate, thus maintaining liquid-vapor equilibrium. Kolditz showed feed pressures maintaining approximately $80 \%$ of the initial feed pressure throughout the nominal thrust duration. The theoretical $I_{S P}$ of hot-water thrusters is the same as that of a steam thruster at the same temperature as long as $100 \%$ of the water evaporates at the throat. However, hot-water thrusters have been empirically shown to experience significant efficiency losses due to incomplete evaporation of the water resulting in an $I_{S P}$ noticeably lower than pure steam; these

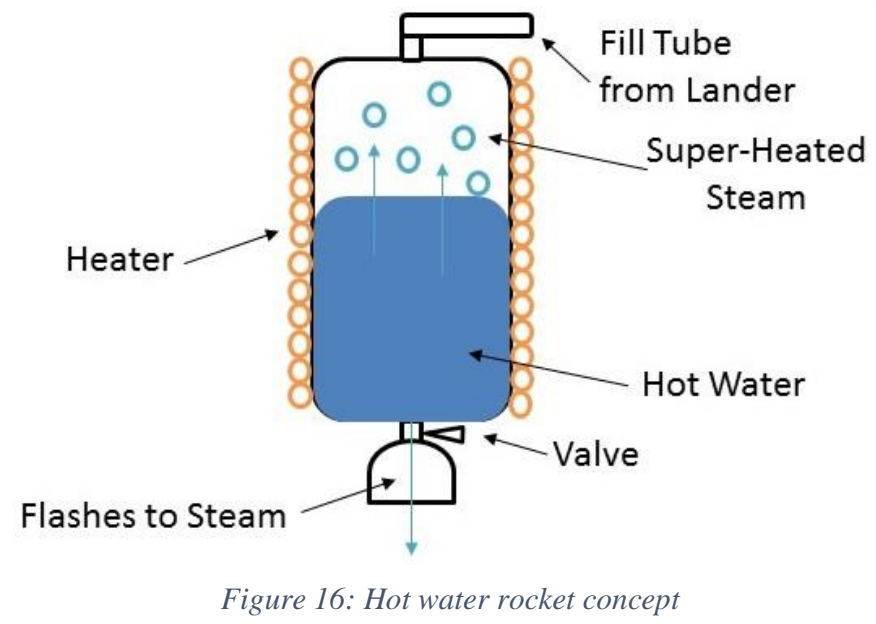

losses also make analytical estimation of the performance challenging and led us to use empirical performance data for our initial calculations. However despite the reduced delivered $I_{S P}$ relative to pure steam, the much higher density achieved by storing the water in liquid form results in a substantial improvement in density $I_{S P}$ compare to a steam thruster and, therefore, significant packaging advantages.

\subsection{Cold Water}

As a propellant, cold water is defined here as that which is stored slightly above its freezing point under pressure. The motivation of such a concept is to achieve
in order to reduce tank volume and empty 
mass. Since the cold water requires a pressurant, chosen in this study to be hot water with a superheated steam head, a second, heated pressurant-tank is necessary as seen in Figure 17. In general, the specific impulse of the cold water as a propellant is very low (maximum of about 20 seconds). During the Phase I study, as discussed in subsequent subsections, it was determined that this substantial reduction in ISP outweighed the benefits of its higher storage densities.

\subsection{Resistojets}

Resistojets rely on a heating element either to heat a cold gas or to evaporate a liquid prior to entry into the thruster nozzle. Resistojets hold the advantage of achieving high specific impulses while maintaining the propellant-density advantage of the cold water concept. Water-based resistojets have been reported by (W. a. Morren 1988) and (T. C. Pugmire

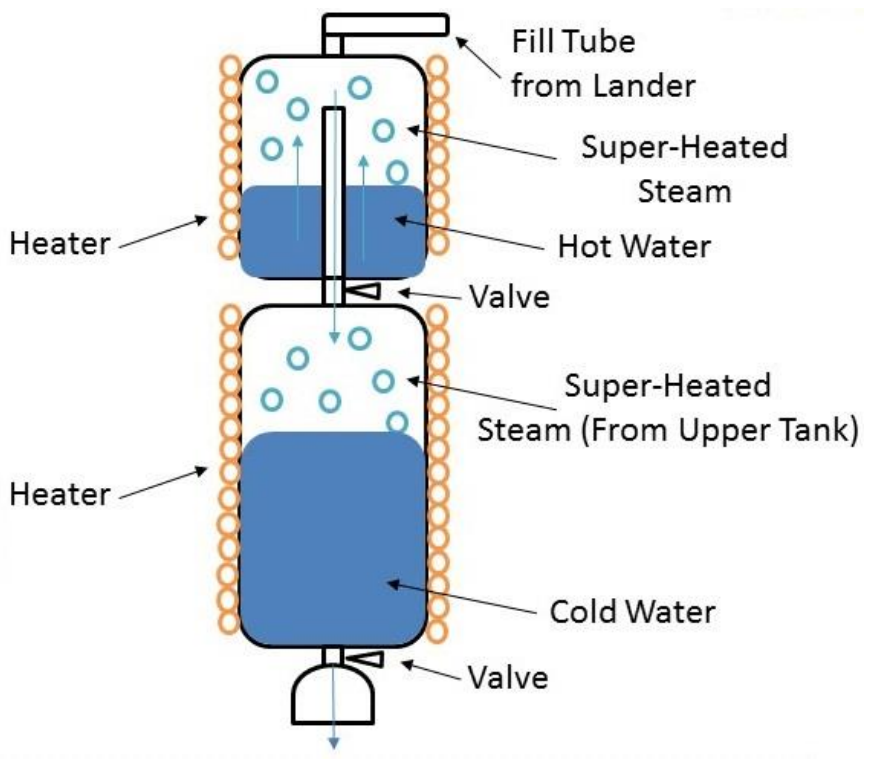

Figure 17: Cold water rocket concept 1986) as one of many possible options for attitude control thrusters. Specific impulses as high as or higher than steam are achievable depending on the operating temperature of the system. High thrust levels, however, are challenging to achieve due to the power requirement associated with the rapid heating of the water at a high mass flow rate. This precipitates the need for a very high thermal power draw from either a thermal mass or ultracapacitor of sufficient capability to support SPARROW. Such

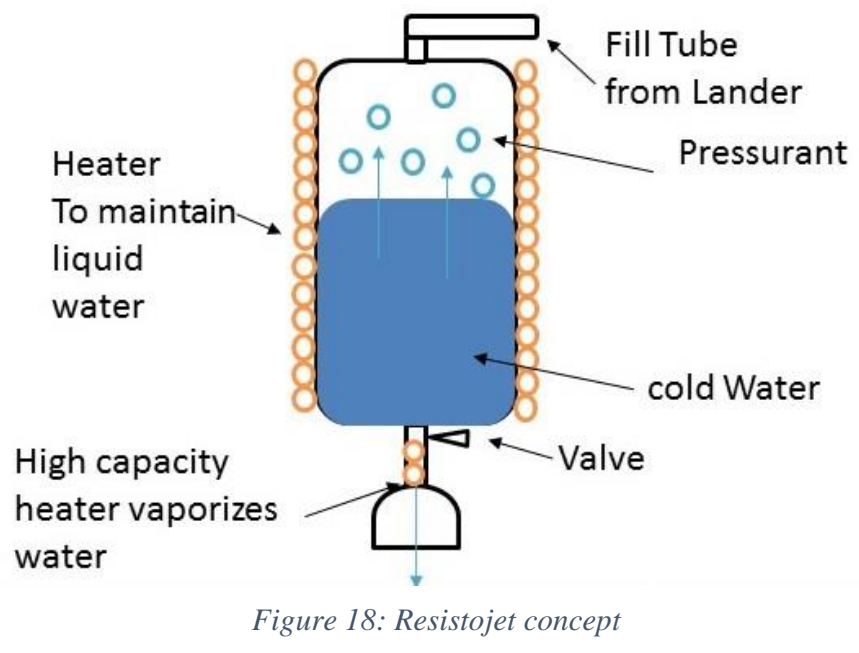
exhausts frequently exceed $2,000 \mathrm{~K}$, likely requiring the nozzles to be housed outside of SPARROW's cage, leaving them vulnerable to impact during hop and tumble activities. 


\subsection{Storable Propellants}

The final concept considered was to bring storable hypergolic propellants such as MMH-NTO from earth, with sufficient reserves for multiple hops. The extra mass of propellant to be launched from Earth and planetary protection concerns make this concept potentially problematic despite its high performance and high TRL. It is included as a point of comparison to the other methods. In the following subsections, performance analysis of these six concepts are introduced.

\subsection{Performance Analyses}

This subsection details a quantitative assessment of each propellant concept. The analyses are analytical and are based on reported values from prior research. Phase I analyses of SPARROW's propulsive and energetic feasibility were performed using several simplifying assumptions: 1) all propellants begin as pure water ice, 2) thermal transfer is adiabatic and isentropic, and 3) propellants behave as ideal gases inside the tank. The objectives of these analyses was to identify the required propellant mass and fuel-tank size and mass needed for each concept, as well as energy requirements. Following the results of the STM, calculations considered round-trip sortie ranges from 1 to 20 $\mathrm{km}$. SPARROW's acceptable dry mass was considered, to the first-order, to be in the range of 5 to $20 \mathrm{~kg}$.

\subsection{Delta- $\mathrm{V}$ and Ballistic Motion}

First-order $\Delta V$ estimates assumed simple ballistic motion over a flat terrain. Other assumptions include the ability to launch at a $45^{\circ}$ angle, which does not take local topographic roughness into account, and an instantaneous impulsive burn. In order to keep the assumption of an impulsive change in velocity reasonable, burn times were restricted to be less than $10 \%$ of the total flight time for each hop. In order to prevent damage to the vehicle landing speed was limited to $15 \mathrm{~m} / \mathrm{s}$, necessitating a deceleration thrust at the end of each hop.

For simple ballistic motion under constant gravity the initial velocity needed to travel a desired distance $D$ is given by Eq. $P_{-}(1)$ :

$$
V=\sqrt{\frac{D * g}{\sin 2 \theta}}
$$

Where the acceleration due to gravity $\mathrm{g}$ on Europa is $1.315 \mathrm{~m} / \mathrm{s}^{2}$. The resulting mission $\Delta \mathrm{V}$ requirements are summarized in Table 1.

Table 1. Summary of mission delta-V requirements

\begin{tabular}{|l|c|c|c|c|c|}
\hline Range (m) & 1000 & 2000 & 5000 & 10000 & 20000 \\
\hline Initial velocity (m/s) & 36 & 51 & 81 & 115 & 162 \\
\hline Maximum altitude (m) & 250 & 500 & 1250 & 2500 & 5000 \\
\hline Travel time (s) & 39 & 55 & 87 & 123 & 174 \\
\hline $\begin{array}{l}\text { Round trip } \boldsymbol{\Delta} \boldsymbol{V} \text { with } \mathbf{5} \mathbf{~ m / s ~ s o f t ~} \\
\text { landings }\end{array}$ & 135 & 195 & 314 & 449 & 639 \\
\hline
\end{tabular}




\subsection{Calculation of $\mathrm{I}_{\mathrm{SP}}$}

For the calculation of $I_{\mathrm{sp}}$, the propellant was assumed to be pure water-ice, with properties taken from the NIST REFPROPS software. Unless otherwise noted, ideal nozzle behavior and $100 \% C^{*}$ and $C_{f}$ efficiencies were assumed.

Steam: The specific impulse of superheated steam at a fixed temperature and pressure can be calculated using the standard equations for $C^{*}$ and $C_{f}$. One challenge is that, assuming a blowdown thruster, the temperature and pressure both decrease as gas is expelled due to expansion in the tank. In order to calculate effective the ISP for superheated steam, a 0dimensional code was written to calculate the impulse of each increment of mass expelled as the tank blows down, and then divide by the initial mass of propellant for an average specific impulse. Isentropic expansion was assumed in the tank. At each step the code checked that the temperatures in the tank and at the nozzle exit were above the saturation temperature and the code was terminated once this condition was not met. After this, significant condensation would begin in either the nozzle or the tank, leading to large efficiency drops to the point where any further thrust produced can be neglected.

Hot Water: While the operating principle of the hot-water propulsion concept is straightforward, its modeling with a sufficient level of details for accurate performance predictions is a challenging task. The difficulties in modeling a hot water propulsion system stem from the need to track phase changes both in the water reservoir and at and near the throat of the propulsion system and the vastly different fluid densities in sub to supersonic flow regimes. While developing a simplified analytical model of the hot-water propulsion concept that could be used for design comparison and sizing was made a priority of the project we initially proceeded using published experimental data. Adirim et al. (Adirim 2006) report an exhaust velocity of $750 \mathrm{~m} / \mathrm{s}$ with an initial tank pressure of $13 \mathrm{MPa}$ and initial temperature of $604 \mathrm{~K}$, if perfect expansion of the nozzle is assumed this translates to a specific impulse of roughly 77 seconds. These values were used for the majority of the design analysis.

Cold water: For pressurized cold water there is no nozzle beyond the throat, and a jet of water is released at a velocity that is dependent upon the feed pressure. Bernoulli's equation was used to estimate the exhaust velocity, which could then be translated to a specific impulse. Using saturated hot water as the pressurant, similar to the hot-water concept, keeps the feed pressure relatively constant for the duration of the burn, and specific impulse at the average of initial and final pressure was used for performance calculations. Once all liquid is expelled, the propellant tank is full of steam still under pressure, which provides additional thrust as it exhausts and this was taken into account for overall average performance.

Resistojet: A water-fed resistojet heats water to steam at a defined temperature during thrust, so would have the same $I_{S P}$ as a steam driven system operating at the same temperature, but without the blowdown effects. $I_{S P}$ was thus calculated using the standard equations for $C^{*}$ and $C_{f}$ with the required properties of steam taken from the NIST database.

Hydrogen + Oxygen and storables: Performance for the Hydrogen/Oxygen combustion concept was based on NASA's CEA code with an O/F of 4 and expansion ratio of 10. For storable propellants we considered Monomethyl Hydrazine (MMH) and Nitrogen Tetroxide (NTO) with an O/F of 1.25 and expansion ratio of 10 .

\subsection{Results of First-Order Calculations}

Table 2 provides a comparison of the six propulsion options for a vehicle empty weight of $20 \mathrm{~kg}$, and a round trip sortie range of $5 \mathrm{~km}$, or five $1 \mathrm{~km}$ hops for the storable option where returning to the lander for refueling is not necessary. 
Table 2. Summary of propulsion performance and propellant requirements

\begin{tabular}{|c|c|c|c|c|c|c|c|c|}
\hline $\begin{array}{c}\text { Propulsion } \\
\text { concept }\end{array}$ & $\begin{array}{c}\text { Initial } \\
\text { tank } \\
\text { pressure } \\
\text { (MPa) }\end{array}$ & $\begin{array}{c}\text { Initial tank } \\
\text { temperatur } \\
\text { e (K) }\end{array}$ & $\begin{array}{l}\text { ISP } \\
\text { (s) }\end{array}$ & $\begin{array}{c}\text { Density } \\
\text { ISP } \\
\left(\mathrm{kg}^{*} \mathrm{~s} / \mathrm{m}^{3}\right. \\
\text { ) }\end{array}$ & $\begin{array}{c}\text { Vehicle } \\
\text { empty } \\
\text { weight } \\
\text { (kg) }\end{array}$ & $\begin{array}{c}\text { Sortie } \\
\text { range } \\
(\mathbf{k m}) \\
\text { with } \\
5 \mathrm{~m} / \mathrm{s} \\
\text { soft } \\
\text { landings }\end{array}$ & $\begin{array}{l}\text { Propellant } \\
\text { mass for } \\
\text { round trip } \\
(\mathrm{kg})\end{array}$ & $\begin{array}{c}\text { Propellant } \\
\text { volume } \\
\text { (liters) }\end{array}$ \\
\hline Steam & 20 & 1250 & 157 & 5520 & 20 & 5 & 4.53 & 129.3 \\
\hline Hot-Water & 13 & 604 & 76.7 & 48909 & 20 & 5 & 10.38 & 16.3 \\
\hline Cold-water & 18 & $630 / 300$ & 20.1 & 16884 & 20 & 5 & 78.7 & 93.7 \\
\hline Resistojet & 1.3 & $1250 / 300$ & 191 & 191000 & 20 & 5 & 3.66 & 3.7 \\
\hline $\begin{array}{c}\text { Hydrogen + } \\
\text { Oxygen }\end{array}$ & 18 & 75 & 425 & 88487 & 20 & 5 & 1.57 & 7.5 \\
\hline MMH+NTO & 2 & 298 & 296 & 331193 & 20 & $5 \times 1$ & 2.47 & 2.2 \\
\hline
\end{tabular}

\subsection{Propellant and tank mass}

The propellant tank mass calculations assume the use of Hastelloy as the material of choice. Tank sizing was performed assuming a spherical shape and a yield safety factor of 1.2. Tank wall thickness used was either that calculated as required to hold internal pressure, or 0.05 inch, whichever was greater. The 0.05 inch minimum thickness serves to maintain structural rigidity.

The minimum wall thickness for a spherical pressure vessel is given by Eq. P_(2):

$$
T_{\text {min }}=\frac{P r}{2 * \sigma_{\text {yield }}}
$$

Where $T$ is the thickness of the tank wall, $P$ is the pressure within the tank, $r$ is the inner radius of the tank, and $\sigma_{y i e l d}$ is the yield stress. Once the wall thickness is determined the volume of the tank walls can be calculated by simple geometry. The necessary mass of tankage for each propulsion system was calculated using the propellant pressures and volumes previously calculated. Table 3 shows a comparison of the results.

Table 3. Propellant tank mass comparison.

\begin{tabular}{|c|c|c|c|c|c|c|c|}
\hline $\begin{array}{c}\text { Propulsion } \\
\text { concept }\end{array}$ & $\begin{array}{c}\text { Initial } \\
\text { tank } \\
\text { pressure } \\
\text { (MPa) }\end{array}$ & $\begin{array}{c}\text { Initial tank } \\
\text { temperature } \\
\text { (K) }\end{array}$ & $\begin{array}{c}\text { Vehicle } \\
\text { empty } \\
\text { weight } \\
\mathbf{( k g )}\end{array}$ & $\begin{array}{c}\text { Sortie } \\
\text { range } \mathbf{( k m )} \\
\text { with } \mathbf{5 ~} \mathbf{~ m} / \mathbf{s} \\
\text { soft } \\
\text { landings }\end{array}$ & $\begin{array}{c}\text { Propellant } \\
\text { mass for } \\
\text { round trip } \\
\mathbf{( k g )}\end{array}$ & $\begin{array}{c}\text { Propellant } \\
\text { Volume } \\
\text { (liters) }\end{array}$ & $\begin{array}{c}\text { Hastelloy } \\
\text { fuel tank } \\
\text { empty } \\
\text { mass (kg) }\end{array}$ \\
\hline Steam & 20 & 1250 & 20 & 5 & 4.5 & 129.3 & 386.0 \\
\hline Hot-Water & 13 & 604 & 20 & 5 & 10.4 & 16.3 & 10.7 \\
\hline Cold-water & 18 & $630 / 300$ & 20 & 5 & 78.7 & 93.7 & 75.0 \\
\hline Resistojet & 1.3 & $1250 / 300$ & 20 & 5 & 3.7 & 3.7 & 1.2 \\
\hline $\begin{array}{c}\text { Hydrogen }+ \\
\text { Oxygen }\end{array}$ & 18 & 75 & 20 & 5 & 1.6 & 7.5 & 5.8 \\
\hline
\end{tabular}


Figure 20 shows the propellant tank masses and volumes vs range for the hot water and Hydrogen-Oxygen combustion propulsion concepts. Note that the $x$ axis, Range, represents the single outbound range and not the full sortie length. The leftmost nts the single outbound range and not the full sortie length. The leftmost $y$ shows propellant tank mass, while the rightmost axis shows tank volume.

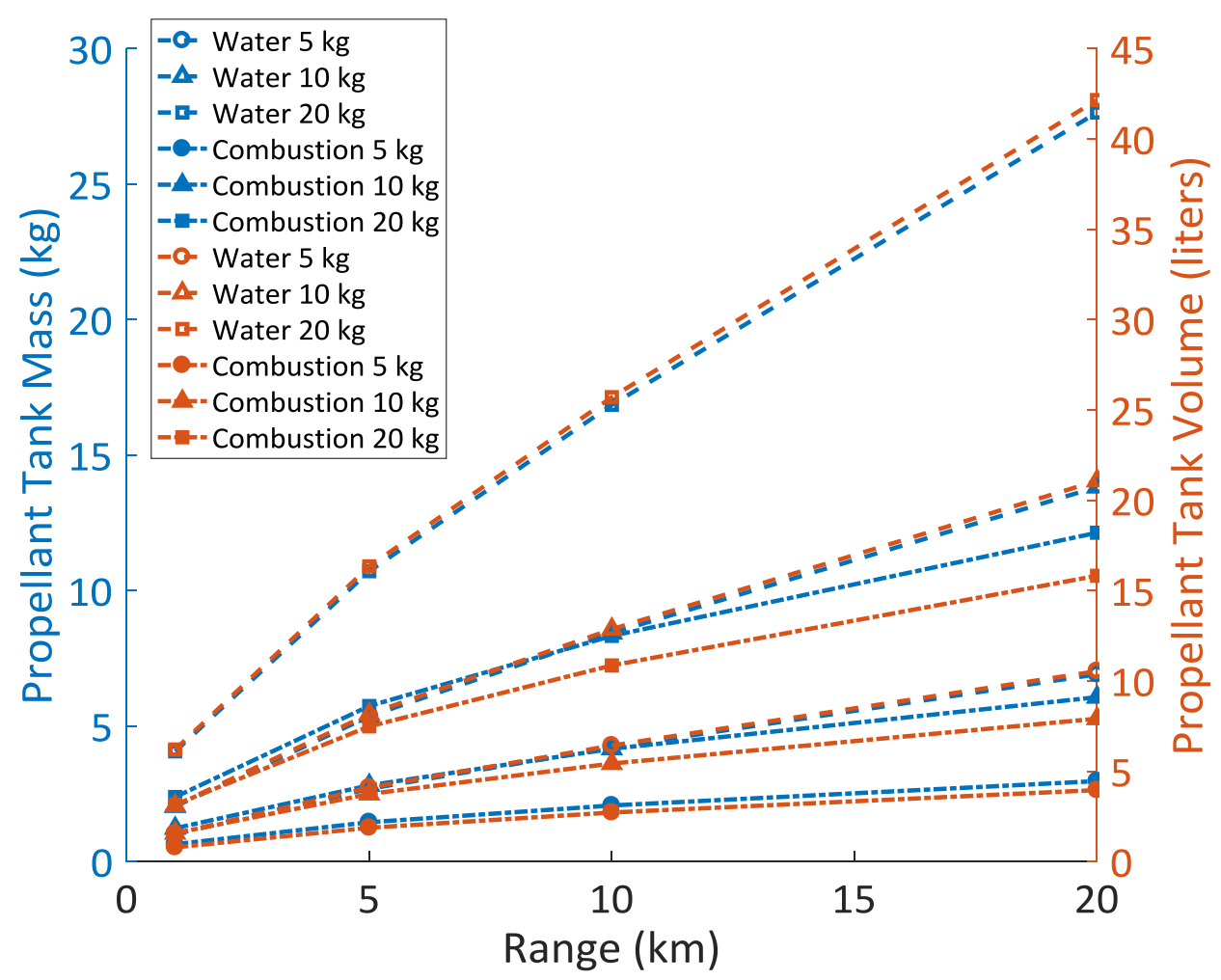

Figure 20. Plot of propellant tank empty mass (blue) and propellant tank volume (red) for multiple sortie ranges at vehicle dry masses of 5 and $20 \mathrm{~kg}$.

\subsection{Propellant Production Energy Requirements}

To calculate the energy required to mine and process the native ice into fuel we assumed that Europan ice starts at $75 \mathrm{~K}$. Heat losses at various stages were neglected at this stage of the analysis but shall be considered in Phase II. For the hydrogen-oxygen combustion option we assumed $75 \%$ efficiency for the electrolysis process. An initial estimate of the energy required to mine ice from the surface was $720 \mathrm{~kJ} / \mathrm{kg}$ based on early experiments at Honeybee. Further energy calculations were performed based solely on heat capacity, enthalpy of fusion, and enthalpy of vaporization from the NIST database. Table 5 provides a summary of the results 
Table 4. Energy and power requirement comparison.

\begin{tabular}{|c|c|c|c|c|c|c|c|c|c|}
\hline $\begin{array}{c}\text { Propulsion } \\
\text { concept }\end{array}$ & $\begin{array}{c}\text { Initial } \\
\text { tank } \\
\text { pressure } \\
\text { (MPa) }\end{array}$ & $\begin{array}{c}\text { Initial tank } \\
\text { temperature } \\
\text { (K) }\end{array}$ & $\begin{array}{c}\text { Vehicle } \\
\text { empty } \\
\text { weight } \\
\mathbf{( k g}\end{array}$ & $\begin{array}{c}\text { Sortie } \\
\text { range } \\
\mathbf{( k m ) ~ w i t h ~} \\
\mathbf{5} \mathbf{m} / \mathbf{s} \text { soft } \\
\text { landings }\end{array}$ & $\begin{array}{c}\text { Propellant } \\
\text { mass for } \\
\text { round trip } \\
\text { (kg) }\end{array}$ & $\begin{array}{c}\text { Energy } \\
\text { required } \\
\text { for } \\
\text { fueling } \\
\text { (MJ) }\end{array}$ & $\begin{array}{c}\text { Energy } \\
\text { required } \\
\text { during } \\
\text { firing } \\
\text { (MJ) }\end{array}$ & $\begin{array}{c}\text { Power } \\
\text { draw to } \\
\text { fuel in } \\
\mathbf{4 8} \text { hours } \\
\text { (W) }\end{array}$ & $\begin{array}{c}\text { Power } \\
\text { draw } \\
\text { during } \\
\text { firing } \\
\text { (MW) }\end{array}$ \\
\hline Steam & 20 & 1250 & 20 & 5 & 4.5 & 26.6 & N/A & 154 & N/A \\
\hline Hot-Water & 13 & 604 & 20 & 5 & 10.4 & 30.0 & N/A & 173 & N/A \\
\hline Cold-water & 18 & $630 / 300$ & 20 & 5 & 78.7 & 128.4 & N/A & 743 & N/A \\
\hline Resistojet & 1.3 & $1250 / 300$ & 20 & 5 & 3.7 & 5.0 & 16.8 & 29 & 0.62 \\
\hline $\begin{array}{c}\text { Hydrogen + } \\
\text { Oxygen }\end{array}$ & 18 & 75 & 20 & 5 & 1.6 & 64.2 & N/A & 371 & N/A \\
\hline MMH+NTO & 2 & 298 & 20 & $5 \times 1$ & 2.5 & N/A & N/A & N/A & N/A \\
\hline
\end{tabular}

Figure 21Figure shows the energy requirements of the hot-water and hydrogen-oxygen combustion propulsion concepts for several combinations of vehicle mass and round trip sortie range.

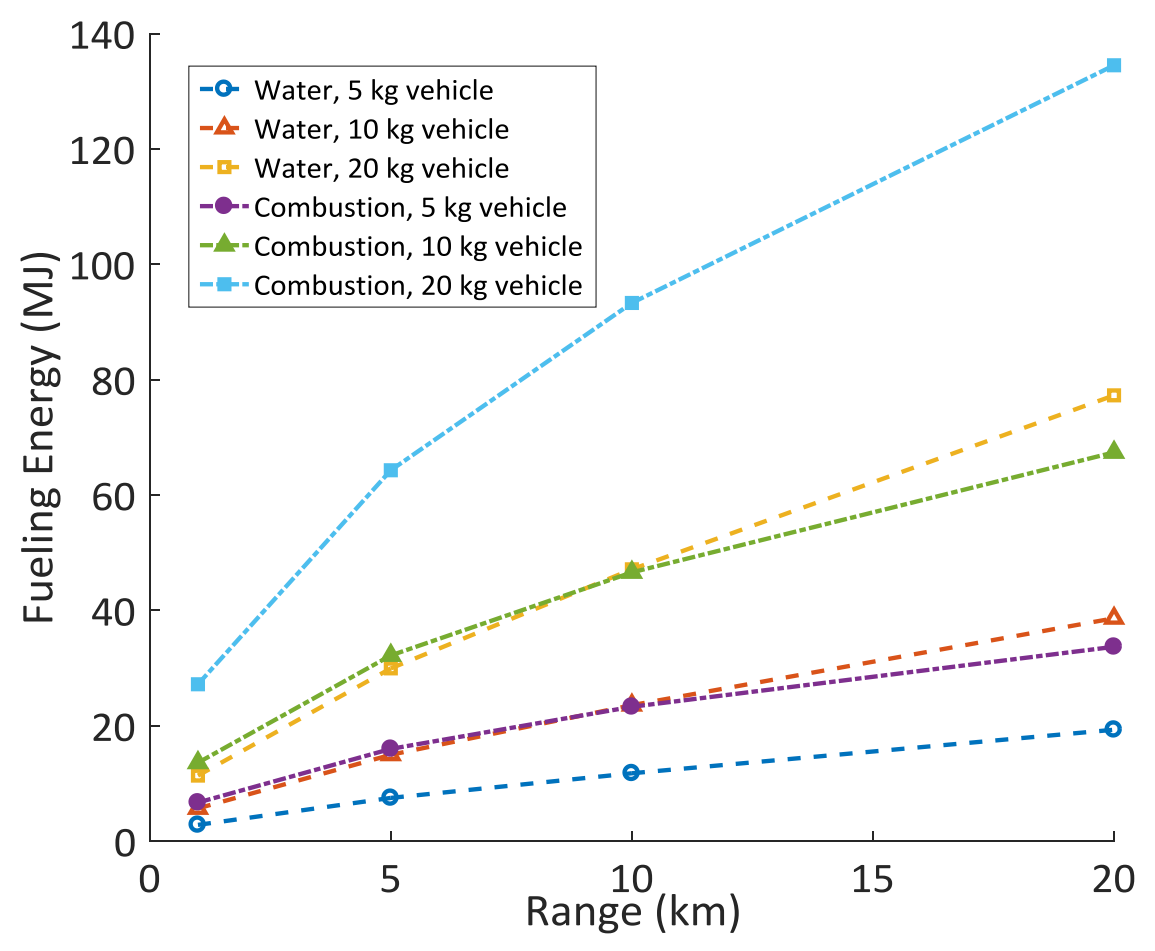

Figure 21. Plot of energy required to fuel vehicle for multiple sortie ranges at vehicle dry masses of 5 and $20 \mathrm{~kg}$.

\subsection{Efficiency Losses due to Cage Drag}

In order to protect the engine and nozzles from impact with terrain features they will likely need to be placed within the protective cage of SPARROW. This means that the exhaust exiting the nozzle will impinge on the cage, creating a requirement that the cage material be able to 
withstand the exhaust temperature, and also causing a loss in engine performance. To estimate these losses the loss in thrust was modeled as being equivalent to the drag that the section of cage interacting with the exhaust plume would experience in a free flow of the same density and velocity. Thus the net thrust is equal to the standalone engine thrust minus the cage drag:

$$
T_{\text {net }}=T-D
$$

Six assumptions were made for the calculation of drag on the cage:

1. The individual elements of the cage are circular in cross section. This is a reasonable shape from a structural perspective and provides a well-known of drag coefficient.

2. The area of the gaps in the cage is significantly larger than the area occupied by the cage bars.

3. The distance between the nozzle exit and the cage is short enough that the flow conditions where it impinges on the cage can be approximated as being the same as the nozzle exit conditions.

4. The flow is entirely gaseous. This would not be completely accurate for several of the propulsion concepts considered, since water condensation is expected to occur as the flow expands in the nozzle, however it is a necessary assumption to simplify the calculation as considering two-phase flow affects in drag would add considerable complexity.

5. The curvature of the cage is small enough relative to the area of impingement that it can be modeled as a flat sheet.

6. The engine nozzle is perfectly expanded. In the near vacuum of Europa a true perfectly expanded nozzle would be impractical, however the pressure difference at the nozzle exit will be low enough that it should not be a major source of error, and it simplifies the results considerably.

The method and equations for estimating drag on a pervious structure were taken from Sighard (Sighard 1951) and were originally developed for estimating drag on dive-brakes and similar aerodynamic surfaces with slats or holes. Drag of pervious surfaces is based on the solidity $\sigma$ defined as:

$$
\sigma=\frac{\text { projected solid area }}{\text { total area }}
$$

Under subsonic conditions the individual elements making up the cage mesh can be treated independently as long as the solidity is less than 0.5 . Therefore with circular cross sections the drag would simply be calculated from the drag coefficient of a long cylinder using only the solid area of the cage where the plume impinges. The drag coefficient $C_{D}$ of a long cylinder is 0.8 . The standard drag equation is:

$$
D=\frac{1}{2} \rho V^{2} S C_{D}=
$$

Where $\rho$ is the fluid density, $V$ is the fluid velocity, and $S$ is the relevant area, in this case the projected solid area of the cage. The thrust of a rocket engine that is perfectly expanded is given by:

$$
T=\dot{M} V_{e}=\rho V_{e}^{2} A_{e}=
$$

Combining equations 5 and 6 and substituting into equation 3:

$$
T_{\text {net }}=T-T\left(\frac{1}{2} C_{D} \frac{S}{A_{e}}\right)=\left(1-\frac{1}{2} C_{D} \sigma\right) T
$$


It can be seen that the loss of thrust, and therefore IsP, will be a constant fraction based only on the $C_{D}$ for the cage elements and the solidity of the cage. Table 5 summarizes the predicted losses for various cage solidities. It can be seen that as long as the solidity of the cage is kept low the losses should remain acceptable. However these calculations have been based on subsonic data. Less information is available for supersonic flow, which a rocket exhaust generally is. However there should still be a solidity below which the cage elements can be treated independently, though it may be lower than the 0.5 solidity that is the cutoff for subsonic flow. At a Mach number of 2 the $C_{D}$ of a long cylinder is 1.25 , and stays relatively constant as Mach number increases from there. Using the supersonic $C_{D}$ in $\left(\mathrm{P}_{-}(7)\right)$ provides the numbers for column 3 in Table 5.

Table 5. Cage Losses

\begin{tabular}{|c|c|c|}
\hline Solidity $\boldsymbol{\sigma}$ & $\begin{array}{c}\text { Percent } \\
\text { thrust/ISP } \\
\text { loss subsonic }\end{array}$ & $\begin{array}{c}\text { Percent } \\
\text { thrust/ISP loss } \\
\text { supersonic }\end{array}$ \\
\hline 0.1 & $4.2 \%$ & $6 \%$ \\
\hline 0.2 & $8.5 \%$ & $13 \%$ \\
\hline 0.3 & $12.7 \%$ & $19 \%$ \\
\hline 0.4 & $17 \%$ & - \\
\hline
\end{tabular}

Phase II research should include more detailed modeling of cage losses, and experimental measurement of cage losses for multiple cage geometries.

\subsection{Propulsion Strategy Selection and Furthered Analyses}

The following criteria were used to evaluate the proposed propulsion methodologies.

1) Feasibility: If the volume or mass of propellant tankage, or the energy requirements for fueling are clearly larger than the scope of the mission can accommodate then the concept is impractical for our purposes.

2) Propellant tank mass: Concepts requiring smaller tank masses, relative to system mass, were given priority.

3) Energy requirements: Concepts with lower propellant production energy cost were preferred, and concepts utilizing the waste heat of a GPHS were given priority.

4) Simplicity: Concepts that are mechanically simpler were preferred.

The relative merits of the 5 proposed propulsion concepts are compared in Table 6 for each of the major selection criteria. Criteria weights range from 1 (low) to 5 (high), and concept scores range from 0 (low) to 5 (high). The hot water rocket concept receives the highest score, with the hydrogen oxygen combustion engine a reasonably close second. 
Table 6. Propulsion Concept Rankings

\begin{tabular}{|c|c|c|c|c|c|c|}
\hline $\begin{array}{c}\text { Propulsion } \\
\text { concept }\end{array}$ & $\begin{array}{c}\text { Feasibility } \\
\text { (Weight: 5) }\end{array}$ & $\begin{array}{c}\text { Propellant } \\
\text { tank mass } \\
\text { (Weight: 3) }\end{array}$ & $\begin{array}{c}\text { Energy } \\
\text { requirement } \\
\text { S } \\
\text { (Weight: 5) }\end{array}$ & $\begin{array}{c}\text { Simplicity } \\
\text { (Weight: 2) }\end{array}$ & $\begin{array}{c}\text { Weighte } \\
\text { d Scores }\end{array}$ & $\begin{array}{c}\text { Rankin } \\
\text { g }\end{array}$ \\
\hline Steam & 0 & 1 & 3 & 4 & 26 & 5 \\
\hline Hot-Water & 4 & 3 & 2 & 4 & 47 & 1 \\
\hline Cold-water & 0 & 1 & 5 & 3 & 34 & 4 \\
\hline Resistojet & 3 & 4 & 1 & 3 & 38 & 3 \\
\hline $\begin{array}{l}\text { Hydrogen } \\
\text { + Oxygen }\end{array}$ & 4 & 3 & 2 & 2 & 43 & 2 \\
\hline
\end{tabular}

The steam and cold-water concepts were removed from consideration early in the project as it became clear that they were infeasible relative to the SPARROW mission concept. At sortie ranges as low as $1 \mathrm{~km}$ the empty propellant tank masses calculated were consistently higher than the assumed dry mass of the entire vehicle so that it was not possible to converge to a workable design. In the case of steam this was due to the low propellant density requiring very highvolume tanks despite the relatively high Isp. Increasing the propellant storage density by increasing the pressure reduced the volume but required pressure high enough that the tank walls became extremely thick, resulting in an overall increase in tank mass. For the cold-water concept the propellant density was much higher, but the specific impulse was so low that very large quantities of propellant were required, again leading to propellant tanks that were larger than the entire vehicle's mass or volume budget.

The resistojet concept was very promising from a propulsion performance perspective, with high density ISP resulting in the lowest tank mass and volume of any of the indigenous propellant options and outscored only narrowly by the storable propellant concept. However, to produce enough thrust for ballistic hops the power draw during thrust generation is extremely high, and we were unable to find a workable power source. Ultracapacitors of sufficient energy capacity would out-mass the vehicle weight, and batteries, while capable of storing the necessary energy for a feasible mass of batteries are not able to deliver it at the required rate. We considered options for reducing the power requirement by pre-heating a large thermal mass that would then transfer heat to the working fluid during thrust generation, however this also adds considerable mass to the system and increases the total energy required. It is possible that with sufficient optimization a feasible solution could be found, however we lacked the resources to pursue this option further with no assurance that we would reach a usable solution.

The hot water concept produces a feasible, if still somewhat high tank mass and volume, and potentially feasible energy requirements. Also, other than the electrical power needed to extract the ice from the surface all energy required for fueling is thermal, allowing use of compact lightweight general purpose radioisotope heating units rather than more massive electrical power sources. Subsequent research in Phase I placed impetus on the further analysis of the hot water rocket system. The concept is quite simple mechanically, but performance analysis is complex due to challenges such as two-phase flow in the nozzle, and significant non-ideal gas behavior. Efforts at analytical modeling of the system and experimental testing of the concept are detailed in the following sections.

The hydrogen-oxygen electrolysis and combustion concept was the next best of the indigenous propellant options after the resistojet, with a required tank mass roughly half that of the hot-water concept. However the energy requirements for the electrolysis are high, and lead to most of the 
required energy being electrical, significantly increasing the required power system mass. The power source could be contained entirely on the lander, since the propellant does not need to be maintained at elevated temperature during sorties, however it could still represent too much mass to allow SPARROW to be a sub-payload of a large mission. This option is also one of the most mechanically complex, between the electrolysis system, which would require contaminants to be removed from the indigenous water before processing, and the complexity of a bipropellant, nonhypergolic liquid rocket engine. We kept the hydrogen-oxygen concept as a second-choice for further analysis, however we focused most of our attention on the hot water concept due to its greater simplicity and lower power requirements.

The storable propellant option is very attractive in terms of performance and removes the need to return to the lander for refueling. Energy requirements are also low, only needing to heat the propellants enough to prevent freezing. This option is also at a very high TRL. The primary argument against bringing propellant from Earth is planetary protection and sample site contamination, which led us to avoid considering this option as anything other than a point of familiar comparison for the lower TRL concepts.

\subsection{Hot-water rocket model}

Since the Hot-Water rocket was one of the most promising concepts evaluated, we desired a way to predict its performance outside the conditions for which experimental data was available in the literature. To that end we sought to develop an analytical model to predict performance of a hot-water system, and to test a hot-water system ourselves for validation of the model.

\subsubsection{Flash Boiling Cycle}

The flash boiling cycle can be described by a triangle, or trilateral cycle. The T-S diagram of this cycle, is shown below in Figure 22Error! Reference source not found..

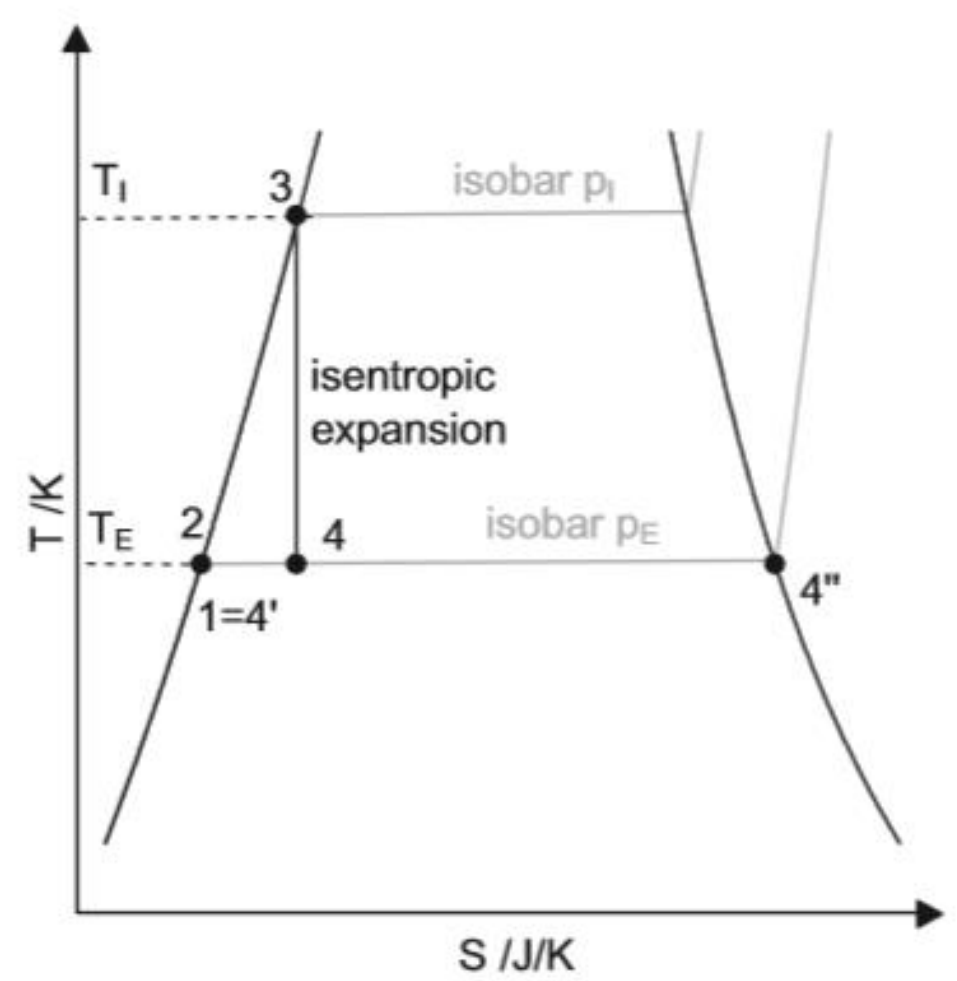

Figure 22. T-S diagram of the ideal triangle cycle with working fluid water. 
In the cycle shown in Figure 22, state 1 represents water at a saturated pressure and temperature. Process 1-2 is work input to isothermally increase the pressure. The pressure at state 2 is the desired saturation pressure of the working fluid. Process 2-3 is heat input required to isobarically increase the temperature of the working fluid so that $T_{3}$ corresponds to the saturation temperature required for $P_{3}$. Process 3-4 is the isentropic work output of the working fluid due to flash boiling expansion.

\subsubsection{Thermodynamic cycle of a hot water rocket}

During Phase I, a thermodynamic model was developed to understand the cycle of a hot water thruster. Ice is emplaced into a closed chamber (the pressure vessel) and a heat source is applied. This causes the temperature of the water to rise until the water reaches the saturation point. The water then rises in pressure and temperature along the saturation curve until it reaches a desired pressure and temperature. The pressure, temperature, and entropy behaviors are shown in Figure 23.
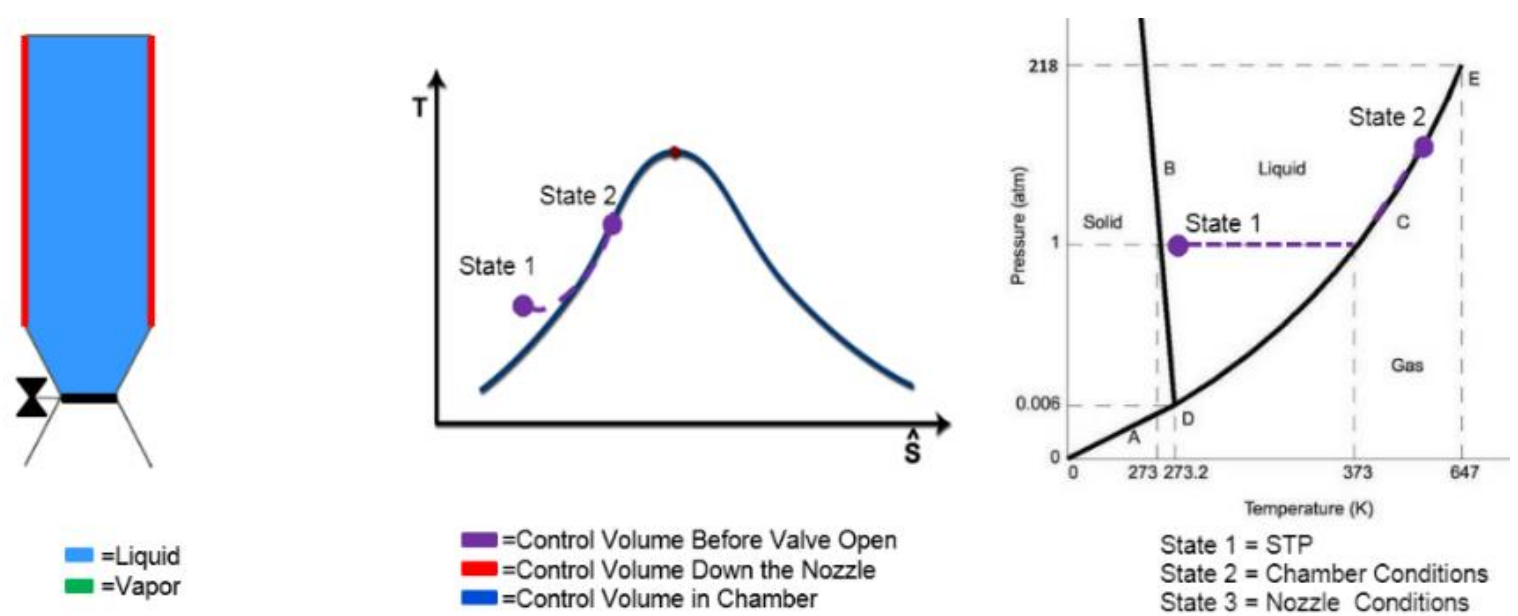

Figure 23: Thermodynamic process of water in a closed container undergoing heat flux

Once the desired pressure and temperature are reached, the chamber is opened to allow water to flow through the nozzle. Opening the chamber creates two control volumes of water: one flowing down the nozzle and an equal volume one in the chamber itself. The control volume of water leaving the chamber to go to the nozzle creates a momentary vacuum in the chamber, which causes a portion of water in the chamber to vaporize in order to restore equilibrium. This vaporization of water in the chamber results in a drop in temperature and pressure. In the nozzle, the control volume of water experiences an area increase. It is assumed that the selected pressure and temperature in the chamber are high enough to choke the flow in the throat, and thus the control volume of water in the nozzle experiences a velocity increase and pressure drop. The control volume of water expands and vaporizes continuously as it travels down the nozzle. Assuming isentropic flow in the nozzle, the conditions of water in the nozzle follow the red path and the conditions of water in the chamber will follow the blue path shown in Figure 24. 

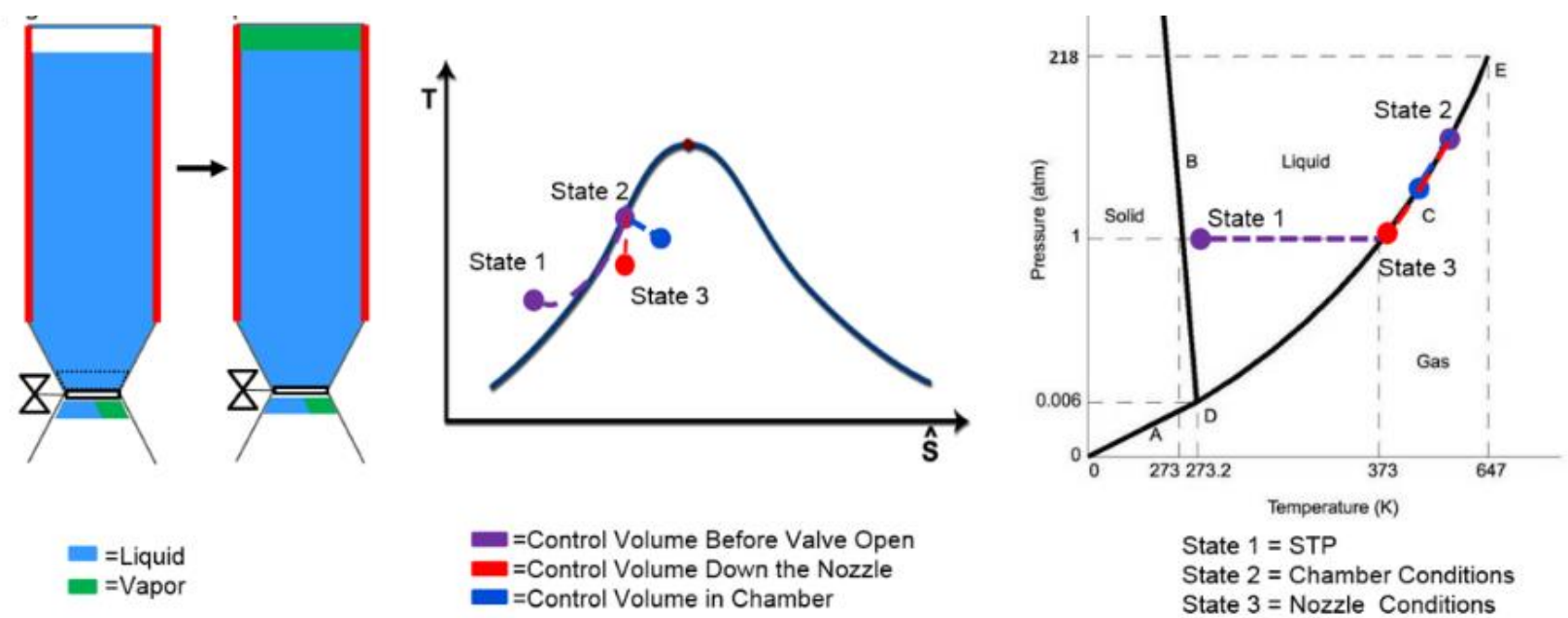

Figure 23: Highly transient thermodynamic process of water being evacuated from test article

Figure 24 shows the conditions of fluid in the chamber and the nozzle as the chamber goes from all liquid to all gas.
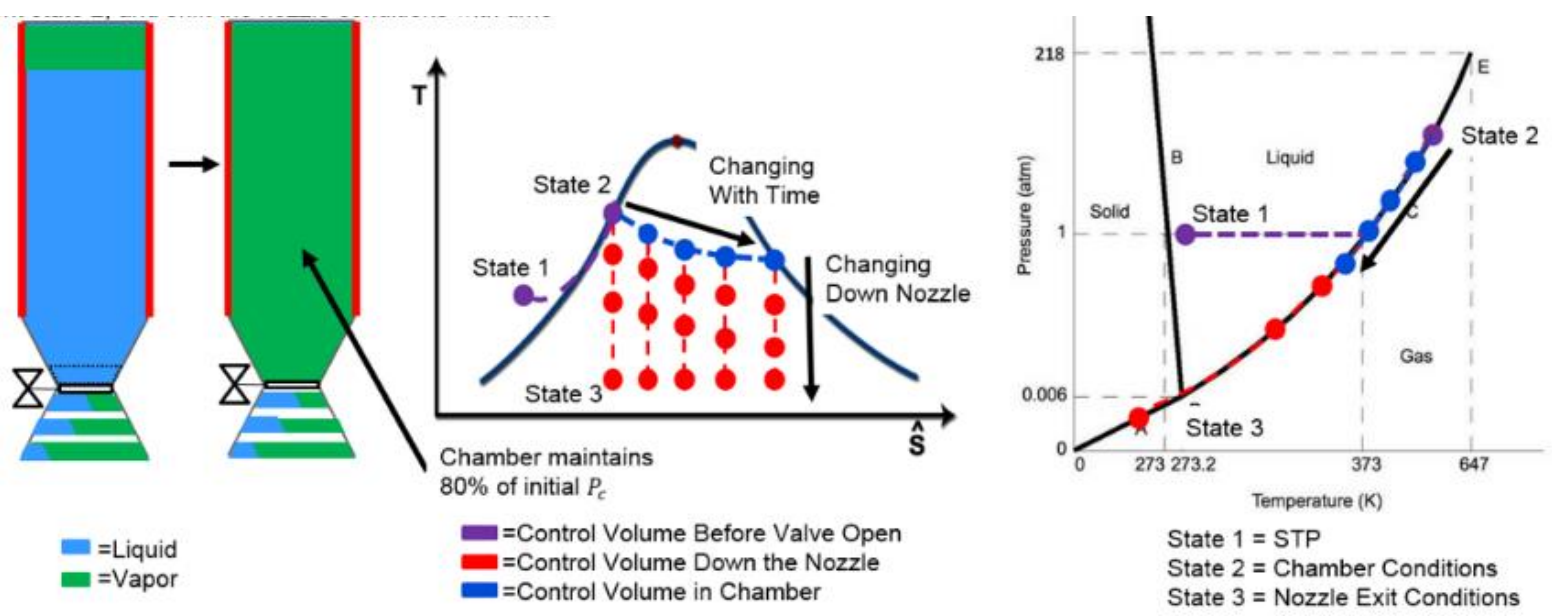

Figure 24. Quasi-steady state process of water being evacuated from test article

As fluid travels down the nozzle, there is mass, momentum, and energy being exchanged between the vapor and the liquid, which means that the vapor and the liquid must be analyzed as two separate control volumes, each with a set of conservation equations. The control volume of fluid in the nozzle is shown in Figure 25. 


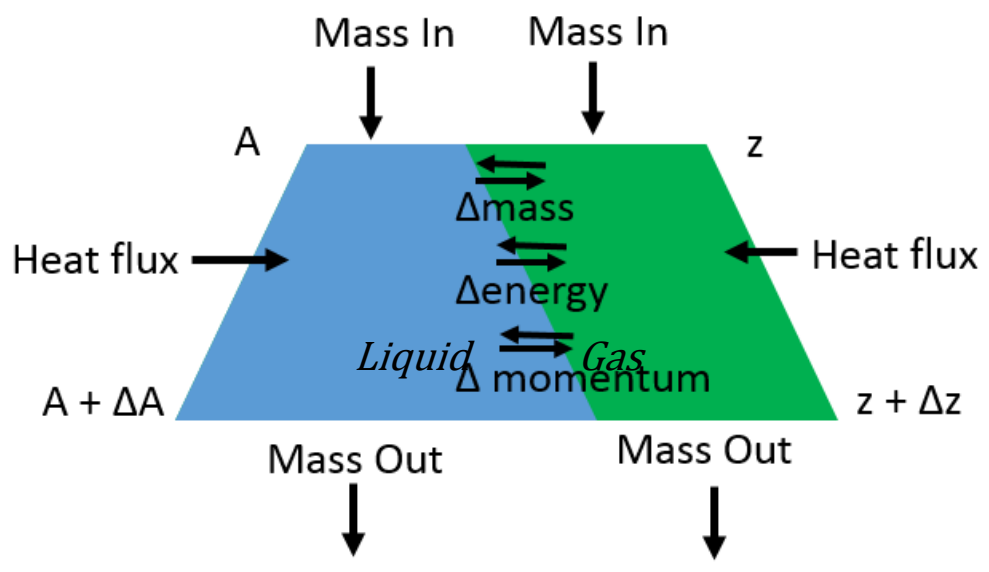

Figure 25. Control volume for two phase flow

The first step in achieving a complete model of a hot water rocket is to get a 1-D steady state model of fluid flow through the nozzle. For this analysis, it is assumed that the gas control volume is in its saturated state, allowing for the enthalpy of the gas and the density of the gas to be a function of pressure only. It cannot be assumed that the liquid is in its saturated state because this would result in a constant vapor quality, which is known to be untrue. Enthalpy as a function of pressure and density as a function of pressure was found by performing a fifth order polynomial curve fit on data taken from the NIST Chemistry Webbook.

A control volume analysis down the nozzle using conservation of Mass, Momentum, and Energy, and vapor quality as a function of enthalpy allow the mass flow rate and average exit velocity to be estimated, which can then yield estimates of the thrust and $I_{S P}$.

\subsection{Hot Water Rocket Testing}

In an effort to verify the results of the previously outlined model, a simple hot water rocket was designed and assembled. The experimental apparatus consists of three main sub-assemblies: a stainless steel tank, a remotely operated run valve, and an expanding nozzle section. The system is described by the plumbing and instrumentation diagram found below in Figure 26. The diagram indicates the relative position of each instrument. Instrumentation for recording pressure and temperature at various points were also included. Pressure measurements were taken from the main chamber and at a point just upstream of the valve and nozzle. Temperature was recorded internally at four points along the center line of the tank along with another measurement just upstream of the valve and nozzle. 


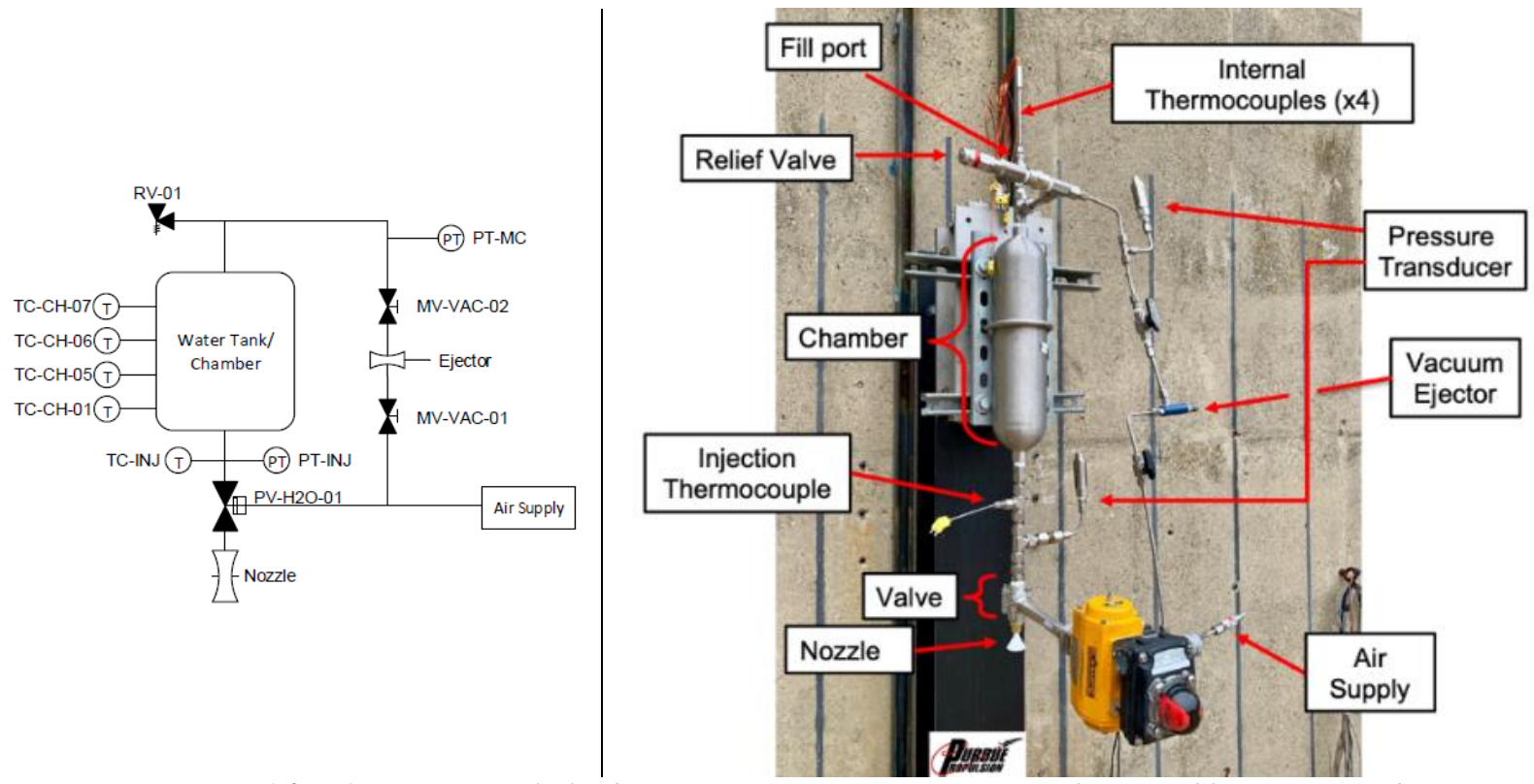

Figure 26. Left: Experimental Plumbing \& Instrumentation Diagram. Right: Assembly on Test Stand

Two nozzles were tested with the same initial tank conditions. Both were modified funnels secured to the test assembly using a compression fitting with a polytetrafluoroethylene (PTFE) ferrule. This ferrule was rated to $250^{\circ} \mathrm{F}$ which made it a potential failure point. The first selected nozzle was made out of PTFE with a throat diameter of 0.201 inch and an expansion ratio of 11.6. This PTFE nozzle was also only rated to $250^{\circ} \mathrm{F}$ which is far lower than the tested water temperature. The nozzle was only subjected to high temperature for the short duration of the test. A glass nozzle was tested second with a throat diameter of 0.222 inch and an expansion ratio 235. An expansion ratio of 3.6 was theoretically required to expand the flow to ambient pressure, so both nozzles were expected to be over expanded. In both tests, the nozzle broke free of the compression fitting. The low-temperature rated attachment mechanism was a likely point of failure, as the PTFE was clearly deformed upon post-test inspection and both nozzles were seen to have some movement in the high speed video prior to nozzle ejection. However, both tests provided a wealth of data.

The thrust time histories corresponding to both tests are presented below in Figure 13 and Figure 14. The thrust levels predicted by the model are also shown. The first test, with the smaller nozzle, lasted approximately 5.3 seconds. The second test, with the larger nozzle, lasted approximately 2.3 seconds. Both tests see a dramatic fluctuation in thrust when the nozzle is lost. In the first test, the nozzle breaks free towards the end of the test at approximately 5.2 seconds after the run valve is opened. In the second test, the nozzle is ejected from the system approximately 0.8 second after the run valve is commanded open. These times were taken from the high speed video footage recorded and compared to the thrust time history. In both cases the thrust achieves a higher value post nozzle ejection, which corresponds to the higher throat area granted to the system. 


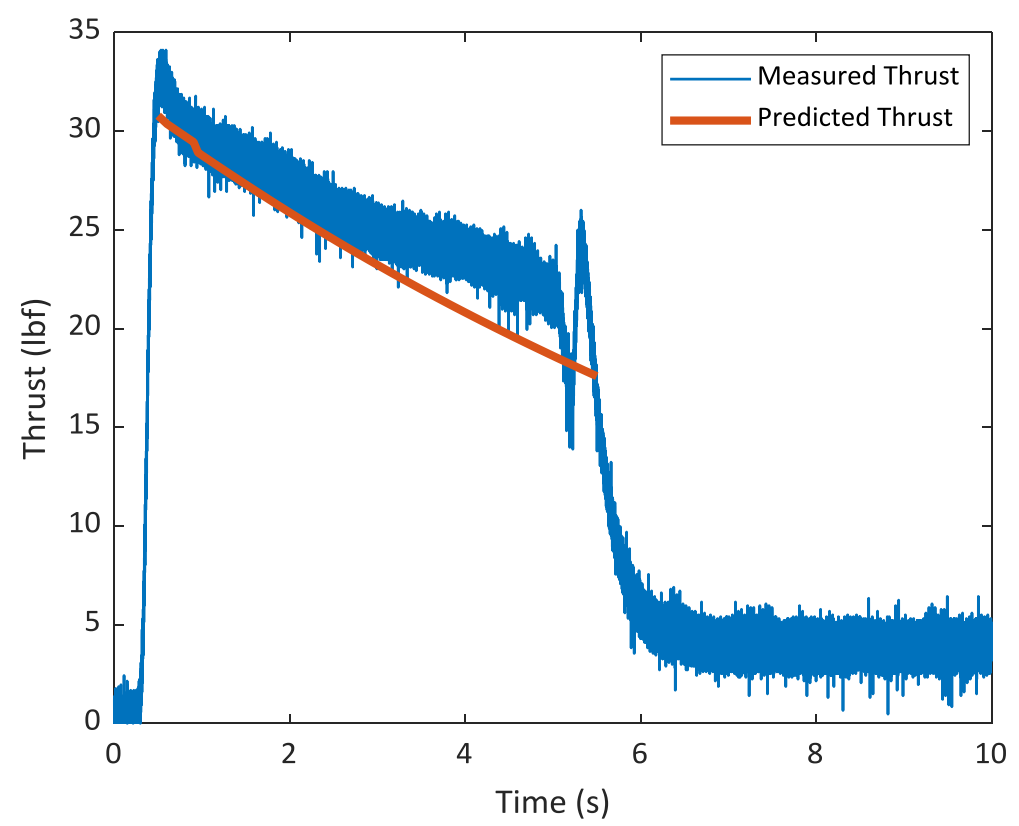

Figure 137. Predicted and measured thrust for test 1, with the Teflon nozzle.

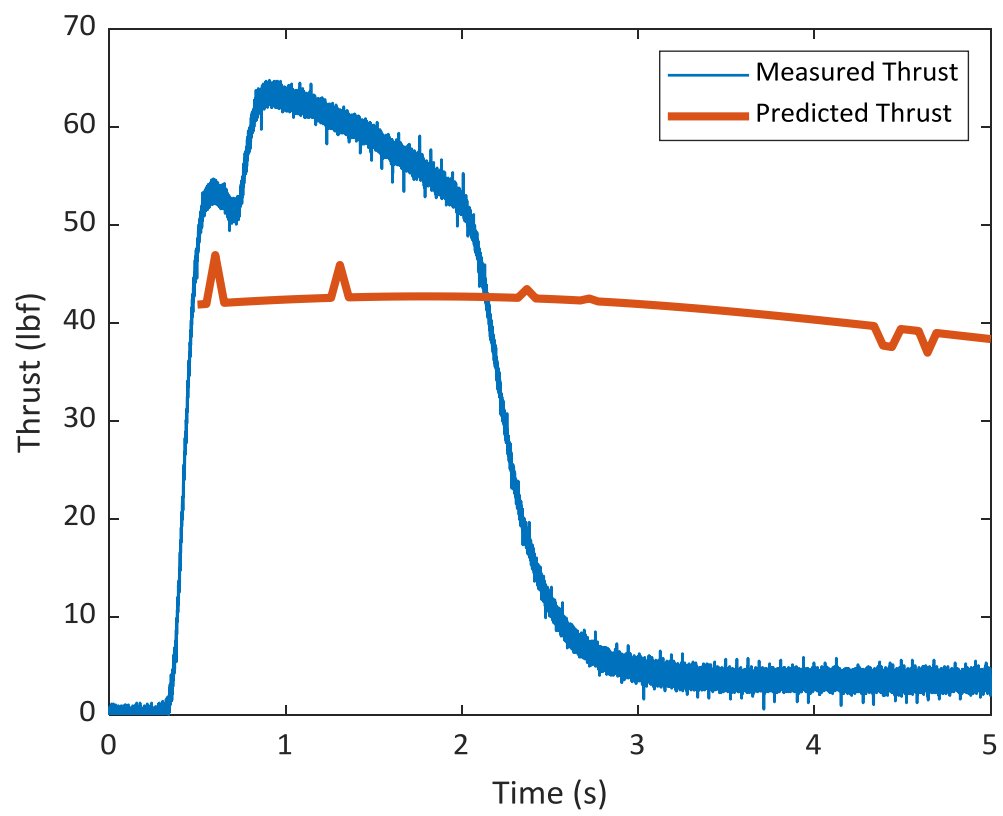

Figure 148. Predicted and measured thrust for test 2, with glass nozzle.

The predicted thrust curve for the second test is not as accurate as that for the first test, this is partly due to how early the glass nozzle failed. Also the model requires a guess at the vapor fraction in the throat to determine the mass flow rate, and this initial guess was provided based on the data from the first test, so the model may be slightly over-fitted to the specific geometry of Test 1 . Further work on the model would need to focus on making it more robust across different geometries. 
Images from the high speed video for the first test at the initial state and just prior to nozzle ejection are shown below. It can be observed that the plume became noticeably narrower as the test progressed.

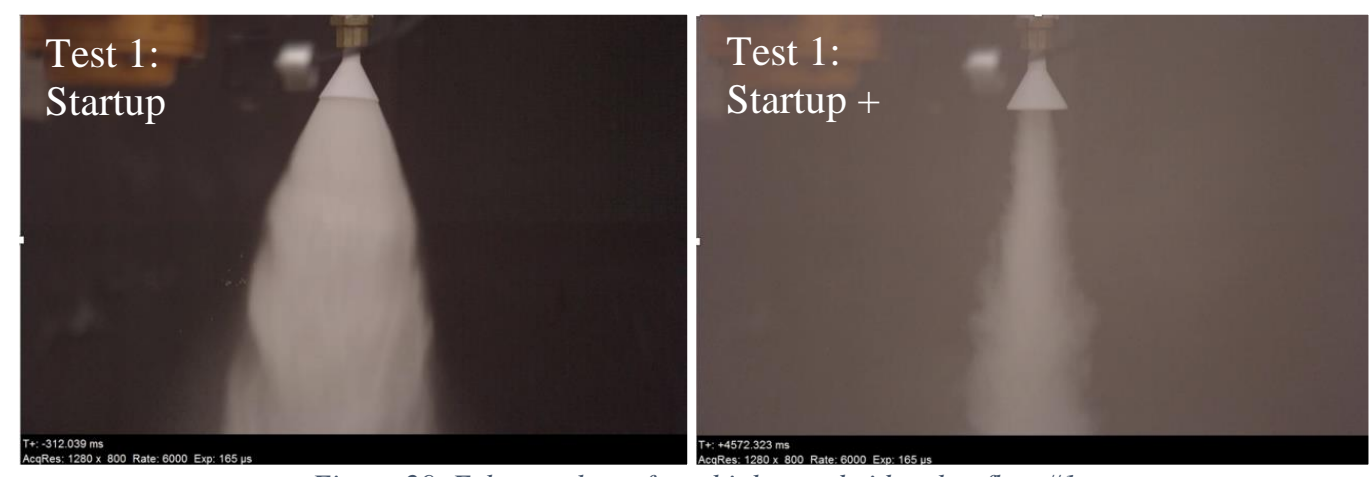

Figure 29. Exhaust plume from high speed video, hot flow \#1

The plume narrowing is much more noticeable in the first hot flow, but the time between the pictures in the first test $(\sim 4.3$ seconds $)$ is much longer than in the second test $(\sim 0.5$ seconds $)$ due to the earlier nozzle failure in the second test, so the chamber pressure decrease is much larger. Even in the short amount of time that the glass nozzle remained intact, a shrinking exhaust plume can be observed in Figure 29.
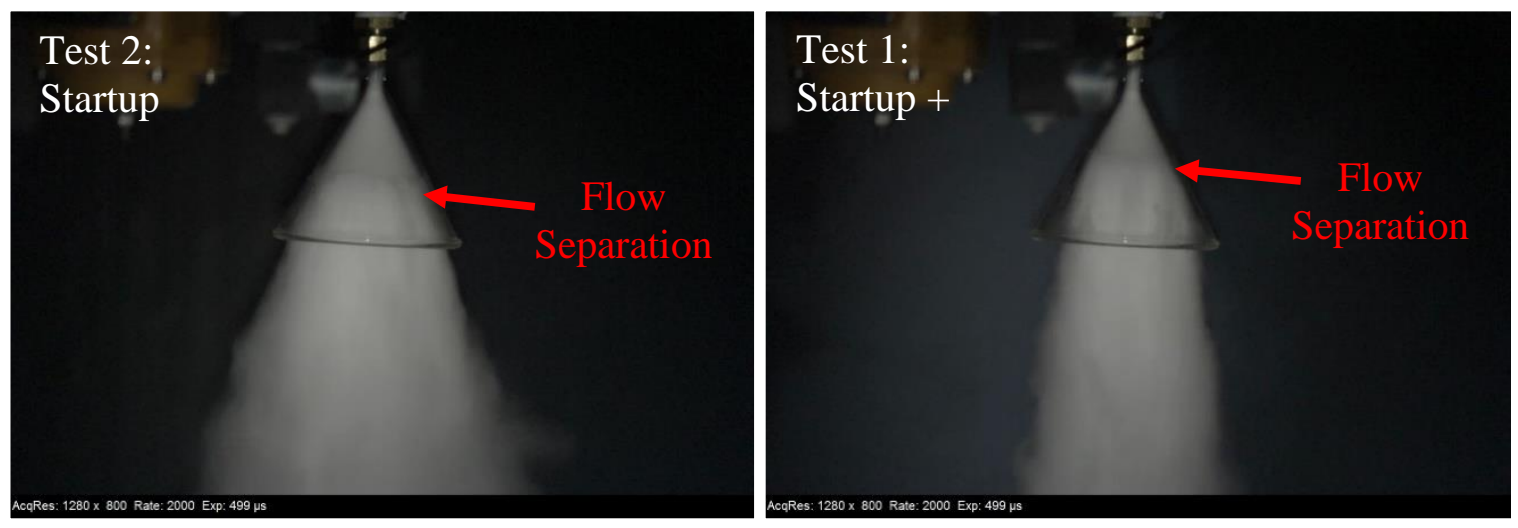

Figure 30. Exhaust plume from high speed video, hot flow \#2

The plume shape change is most likely due to the large decrease in chamber pressure that occurs, which would indicate that the flow should need less expansion to reach a state where flow separation from the nozzle occurs. The optical access of the glass nozzle from the second test provides a view of the flow separating from the nozzle and how the location of separation moves upstream over time. The glass nozzle did not withstand the harsh conditions of the high temperature, high pressure exhaust, but it did provide a unique look into the internal flow characteristics of the nozzle.

\subsection{Effects of Ice Impurities on Propulsive Efficiency}

All preceding analyses assumed relatively pure water ice as input. However as discussed with regards to materials selection, it is expected that Europan ice will have a variety of impurities including sulfuric acid and various salts. The change in thermophysical properties caused by the addition of impurities will have an impact on engine performance which needs to be addressed. The main candidate concept still under consideration is the saturated hot-water rocket. 
Expanding the analytical model that was developed for pure water to include saltwater of varying concentrations was well beyond the scope of phase I, however some general trends can be estimated simply by looking at the changes in properties.

Since the hot-water concept operates on the saturation curve for water the first property to examine is how the saturation pressure for a given temperature varies with salt concentration. Dittman provides the following table and chart of saturation pressures as a function of temperature and weight percent salt. The higher the salt concentration the lower the saturation pressure for a given temperature, this will reduce the mass flow rate through the engine, and therefore the thrust, since it is a pressure fed system. The saturation pressure coefficients are provided by equation P_8.

$$
P_{\text {sat-brine }}(T)=a 1 * P_{\text {sat }}(T)=
$$

The saturation pressure coefficients for brines of several salt concentrations are given in Table 7 and a plot of the resulting saturation curves are shown in Figure 31.

Table 7. Saturation Pressure coefficients

\begin{tabular}{|c|c|}
\hline Wt. \% salt & a1 \\
\hline 5 & 0.969 \\
\hline 10 & 0.934 \\
\hline 15 & 0.894 \\
\hline 20 & 0.847 \\
\hline 25 & 0.794 \\
\hline
\end{tabular}

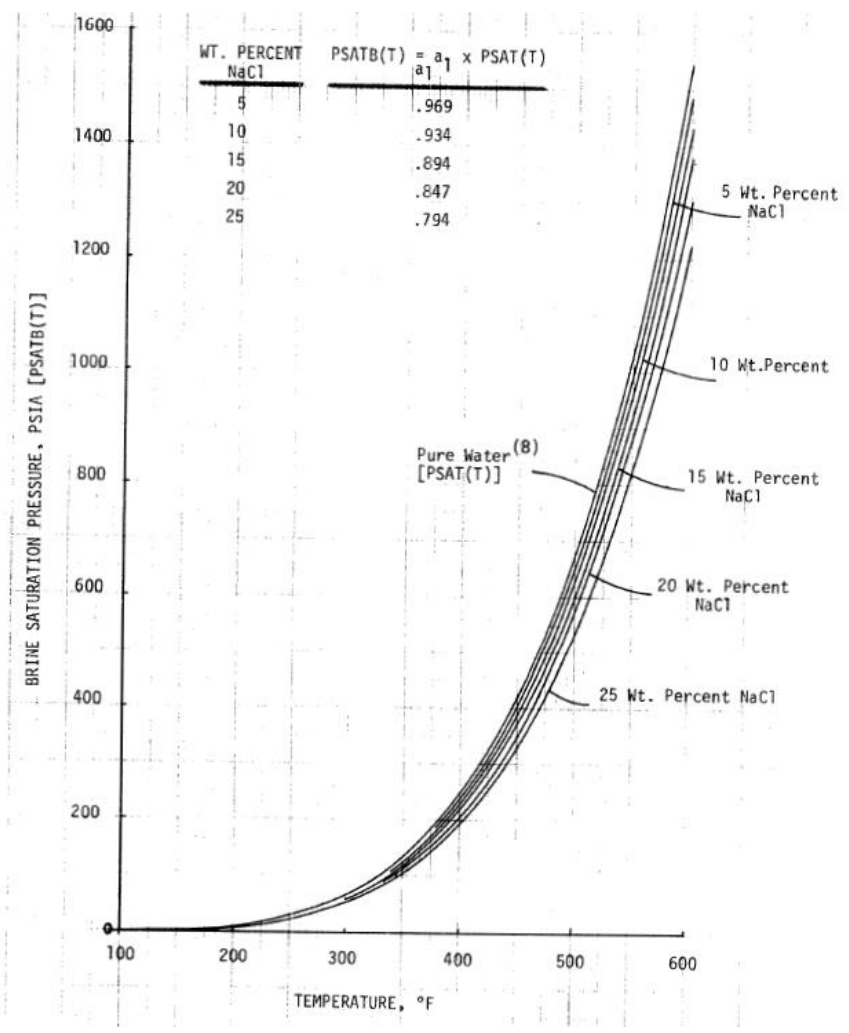

Figure 31: Calculated brine saturation pressure curves from data for dissolved $\mathrm{NaCl}-\mathrm{H}_{2} \mathrm{O}$ solutions. 
Density is also affected by salt concentration, with the density increasing as the salt concentration increases, Figure 32 shows density curves for liquid brines with varying temperature.

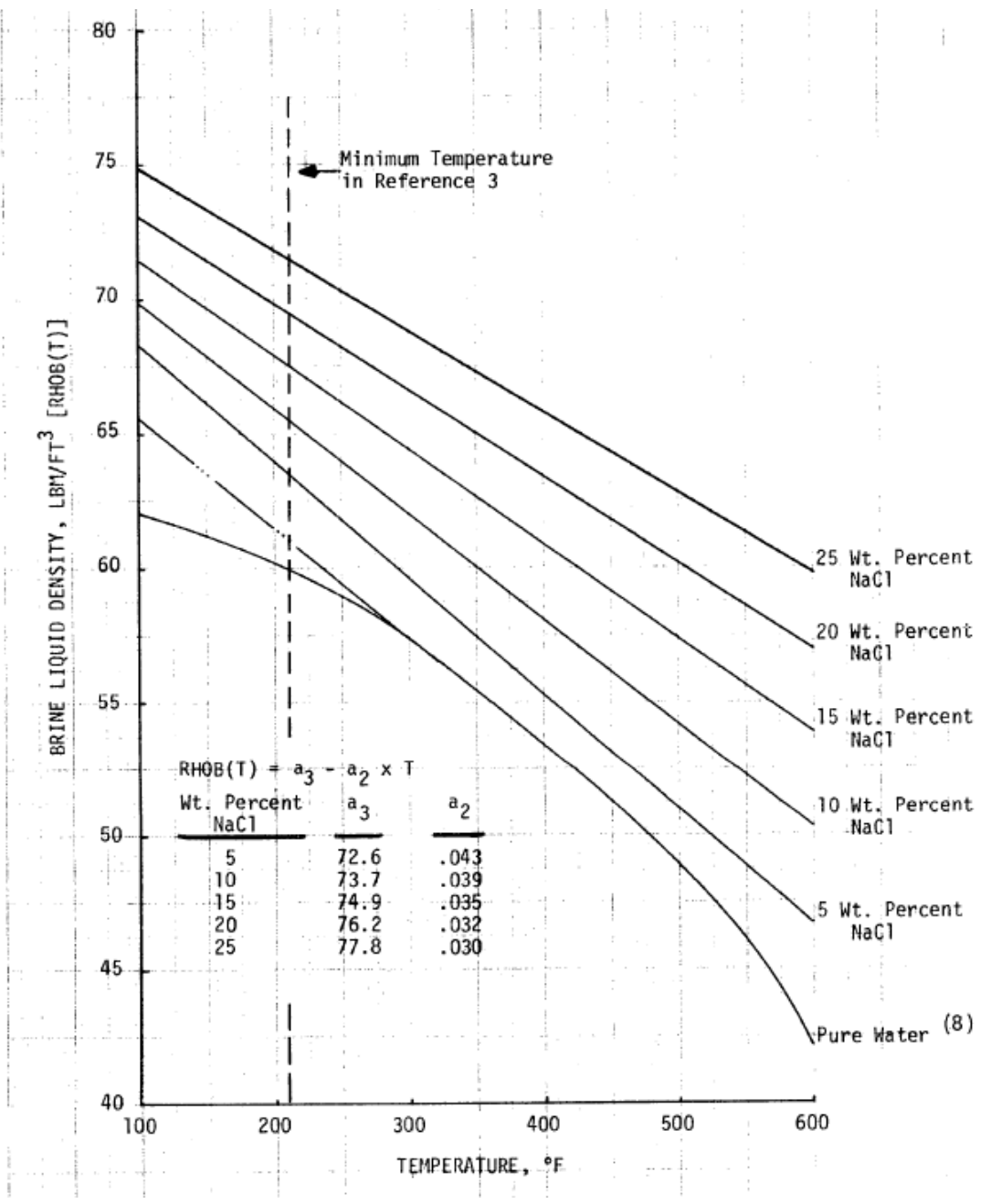

Figure 32: Calculated Brine liquid density curves from Data for Dissolved $\mathrm{NaCl}-\mathrm{H}_{2} \mathrm{O}$ Solutions.

Higher salt concentration increases the liquid density, however the percent change in saturation pressure is larger, so since mass flow through an orifice is proportional to both feed pressure and liquid density overall the mass flow will decrease for a given engine geometry. To compensate for these effects a larger throat diameter would need to be used than in a system designed for pure water, with the throat area inversely proportional to pressure and density to maintain a roughly constant flow rate. However, with the actual concentration and composition of impurities in the ice on Europa unknown the vehicle control system will need to be able to accommodate significant variation in mass flow rate, and therefore thrust, depending on the actual density and saturation pressure of the extracted propellant.

Impurities will also affect the IsP of the system. The major change will likely be caused by differences in the vapor fraction in the nozzle, however modeling the nozzle vapor fraction is challenging even for pure water, and incorporating the effects of impurities would be a major 
undertaking. If it is assumed that the vapor fraction is not affected by the impurities then the only change in engine efficiency will be caused by the change in average molecular weight of the exhaust. Table 8 summarizes the effects of molecular weight using $\mathrm{NaCl}$ salt as an example once again.

Table 8. ISP loss with $\mathrm{NaCl}$ weight percent

\begin{tabular}{|c|c|}
\hline $\begin{array}{c}\text { NaCl } \\
\text { wt. \% }\end{array}$ & $\begin{array}{c}\text { ISP \% of } \\
\text { pure water }\end{array}$ \\
\hline 0 & 100 \\
\hline 5 & 98.3 \\
\hline 15 & 94.7 \\
\hline 25 & 90.9 \\
\hline
\end{tabular}

\subsection{Heat Losses}

For a first order estimate of how fast the propellant would cool after being brought up to temperature we assumed that the propellant tank could be sufficiently isolated from the surface that conduction was negligible, since a conduction analysis would depend on details of the vehicle structure that have not been specified at this stage of the design. With the near vacuum atmosphere of Europa this leads to radiation being the only relevant means of heat transfer. Since the propellant tank is thin-walled and metal we also assumed no significant thermal gradient in the tank wall, such that the surface temperature of the propellant tank is the same as the desired storage temperature of the propellant.

The other necessary parameters for radiative heat loss calculations are the emissivity of the material, and the external temperature. The emissivity of Hastelloy is reported as 0.88 , which produces a fairly high loss rate, therefore gold-plating the tanks was considered as an insulating mechanism, the emissivity of gold-plated Hastelloy is 0.028 . A vacuum temperature of $3 \mathrm{~K}$ was used to give an upper bound, although the lower half of the tank would actually be attempting to reach equilibrium with the $75 \mathrm{~K}$ surface.

Only the hot-water propulsion system was considered for heat-loss rates, as the hydrogen and oxygen for the combustion option would be stored at ambient temperature to improve density and avoid ongoing energy expenditure, and other options had already been deemed impractical by this stage of the project. 


\begin{tabular}{|c|c|c|c|c|c|c|}
\hline $\begin{array}{c}\text { Propulsion } \\
\text { concept }\end{array}$ & $\begin{array}{c}\text { Initial tank } \\
\text { temperature } \\
(\mathrm{K})\end{array}$ & $\begin{array}{c}\text { Vehicle } \\
\text { empty } \\
\text { weight } \\
\text { (kg) }\end{array}$ & $\begin{array}{c}\text { Sortie } \\
\text { range }(\mathbf{k m}) \\
\text { with } 5 \mathrm{~m} / \mathrm{s} \\
\text { soft } \\
\text { landings }\end{array}$ & $\begin{array}{c}\text { Power } \\
\text { draw } \\
\text { to fuel } \\
\text { in } 48 \\
\text { hours } \\
(\mathrm{W})\end{array}$ & $\begin{array}{c}\text { Heat loss } \\
\text { (Hastelloy) } \\
(\mathrm{W})\end{array}$ & $\begin{array}{l}\text { Heat loss } \\
\text { (gold } \\
\text { plated) }(W)\end{array}$ \\
\hline \multirow{9}{*}{$\begin{array}{c}\text { Hot } \\
\text { Water }\end{array}$} & \multirow{9}{*}{604} & 5 & 1 & 16 & 429 & 14 \\
\hline & & 5 & 5 & 43 & 822 & 26 \\
\hline & & 5 & 10 & 68 & 1110 & 35 \\
\hline & & 10 & 1 & 33 & 683 & 22 \\
\hline & & 10 & 5 & 87 & 1303 & 42 \\
\hline & & 10 & 10 & 137 & 1763 & 56 \\
\hline & & 20 & 1 & 66 & 1084 & 35 \\
\hline & & 20 & 5 & 173 & 2068 & 66 \\
\hline & & 20 & 10 & 273 & 2797 & 89 \\
\hline
\end{tabular}

\section{Controls and Localization}

In order to simplify the hopping control and eliminate reliance on in-flight attitude control, Phase I analyses adopted a "point-andshoot" hopping control, whereby SPARROW adjusts its orientation on the surface such that it is pointing along the desired hop direction, executes a single burn at maximum thrust to follow a ballistic trajectory. Additionally, we considered two potential "modes" for SPARROW's hopping control. A direct-hop control policy attempts to intercept the target location in a single parabolic trajectory, requiring a large initial burn for takeoff and one burn for soft-landing. Alternatively, a multi-hop control policy hops directly towards the goal, but with limited speed per hop so as to avoid the need for retroburns (i.e., just passive landing/bouncing). The single-hop control strategy is more fuel-efficient, but it is susceptible to a mission-ending surface impact if the retro-burn fails. On the other hand, the multi-hop policy avoids this potential risk, but at the cost of poorer fuel efficiency and less tolerance to intermediate terrain roughness between the lander and target location.

Table 11: Comparison of direct hop and multi-hop architecture benefits

\begin{tabular}{|l|l|l|}
\hline & Direct Hop & Multiple small hops \\
\hline $\begin{array}{l}\text { Control } \\
\text { Policy }\end{array}$ & $\begin{array}{l}\text { Execute a single hop to target } \\
\text { location, which requires a retro-burn } \\
\text { for soft landing }\end{array}$ & $\begin{array}{l}\text { Take multiple smaller hops towards } \\
\text { target which do not require retro burns }\end{array}$ \\
\hline Pros & $\bullet \quad$ More fuel-efficient & $\bullet$ Guaranteed safe landing \\
\hline
\end{tabular}




\begin{tabular}{|c|c|c|}
\hline & $\begin{array}{l}\text { - Higher vantage point for } \\
\text { localization } \\
\text { - Less susceptible to irregular } \\
\text { terrain irregularities }\end{array}$ & $\begin{array}{l}\text { - Possible intermediate science } \\
\text { opportunities }\end{array}$ \\
\hline Cons & $\begin{array}{l}\text { - Failed retro-burn would end } \\
\text { mission }\end{array}$ & $\begin{array}{l}\text { - Accumulation of localization } \\
\text { errors } \\
\text { - } \begin{array}{l}\text { More susceptible to irregular } \\
\text { terrain irregularities }\end{array}\end{array}$ \\
\hline
\end{tabular}

While SPARROW's mobility paradigm of large ballistic trajectories is much more terrain agnostic than traditional surface mobility, it is still subject to a series of landing bounces on the largely unknown surface. Moreover, errors in hop execution (e.g., speed and direction) may induce significant trajectory variability. In order to quantify the resulting errors in landing precision and the need for subsequent corrective hops, we developed a simulation environment in which these various sources of uncertainty can be injected arbitrarily, and a large batch of Monte Carlo simulations can be run to observe the statistical variability. As discussed in Section 4.2, terrain maps of Europa at the rover scale are not available and are unlikely to be available even prior to landing. As a Europa surface analog, we mapped a 50m region of glaciated ice flow in Alaska, which was used to create a 1 million-facet triangular surface mesh (see Fig. 33). While there is likely to be significant differences in the structure and geological processes between Europan and terrestrial terrain, this mesh does exhibit many features (e.g., pits and fractures) that we observe at a larger scale on Europa. Thus, for our simulations we scale this mesh up about 300x so that it better matches the resolution of our images and so that we can simulate a $1 \mathrm{~km}$ sortie. Surface irregularities below the facet scale are roughly captured by randomizing the surface normal upon impact. 

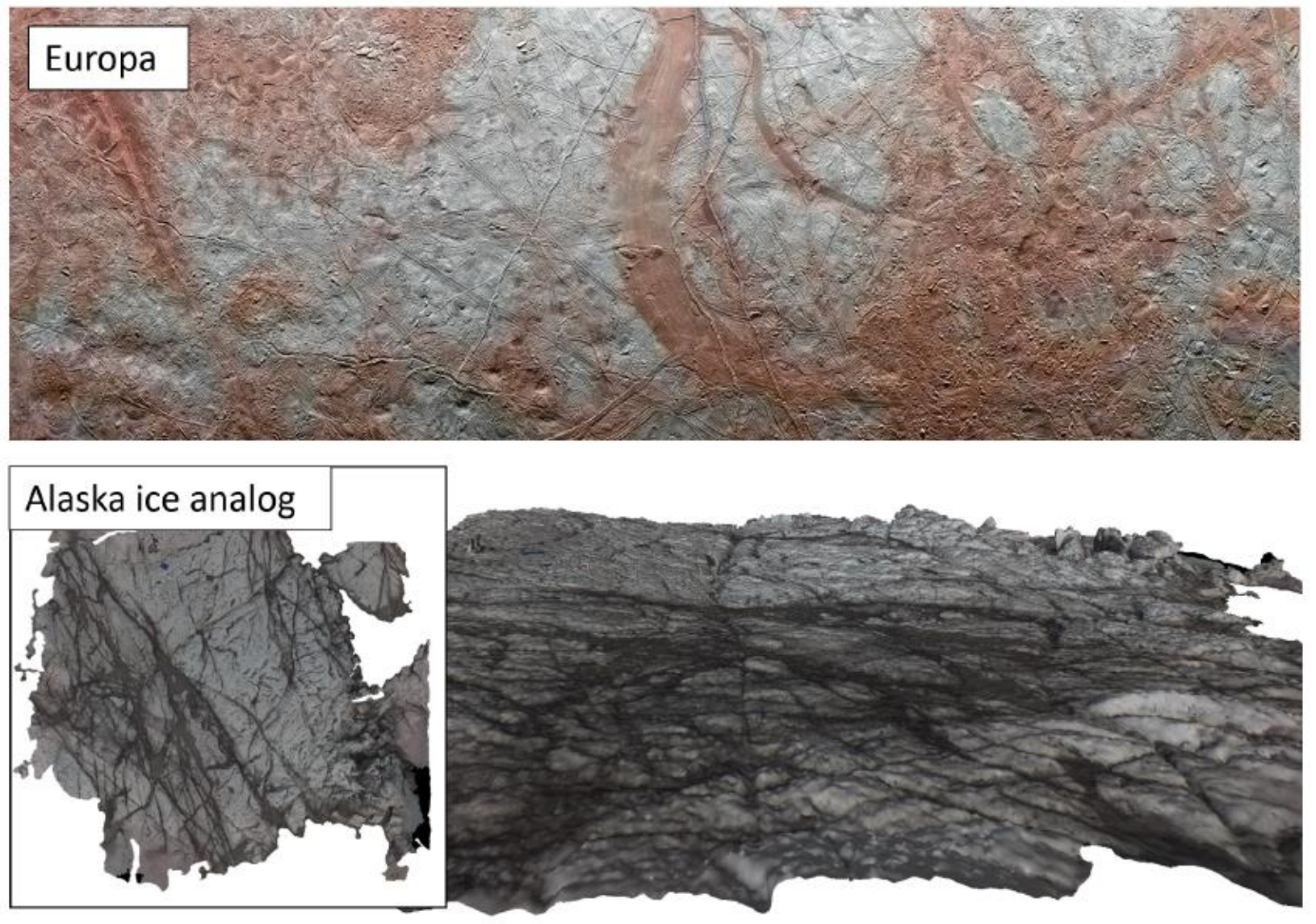

Figure 33: Comparison of Europan surface as imaged by Galileo and the shape model of an Alaskan ice sheet used as an analogue for simulations.

Each simulation consists of a series of hops, whereby the desired hop velocity vector is recomputed based on SPARROW's position (assuming perfect estimation) relative to the target region each time the rover comes to rest on the surface. Once the target region has been reached, this process is repeated as SPARROW returns to the lander. SPARROW is modeled as a particle in a constant gravity field $\left(\mathrm{g}=1: 315 \mathrm{~m} / \mathrm{s}^{2}\right)$. The initial hop velocity is randomized according to the expected control uncertainty (e.g., an unbiased Gaussian) and the rebound velocities are also randomized. For this case study, we assume a hop pointing uncertainty of $5^{0}$, a speed uncertainty of $5 \%$, a mean surface restitution of $0: 6$ with standard deviation of $0: 1$, and a rebound angle reflected about a randomized normal vector with $10^{\circ}$ about the facet normal. Also, we assume an IsP of $75 \mathrm{~s}$, a dry mass of $20 \mathrm{~kg}$, and a $15 \mathrm{~m} / \mathrm{s}$ velocity limit for the multihop sorties. The results of 1000 Monte Carlo simulations for both control policies are summarized in Fig. 34, and a few example trajectories for each policy are shown in Fig. 35. Box plots correspond to the maximum, minimum, 25 th and $75^{\text {th }}$ percentile fuel cost for each sortie distance. The solid lines correspond to the first order prediction for each control policy (i.e., no control errors or bouncing). As expected, the landing errors induced due to control errors and bouncing require corrective hops for the single-hop policy, resulting is slightly increased fuel consumption for a given sortie distance (about 20\%). On the other hand, the simulated multi-hop sorties actually outperform the first-order predictions (i.e., reduced fuel usage), likely due to the fact that the bounces, on average, yield forward progress. Overall, with $10 \mathrm{~kg}$ of fuel, these results suggest 
that SPARROW has a maximum range (with a $90 \%$ success rate) of about $1.6 \mathrm{~km}$ for the multihop control policy and $2.5 \mathrm{~km}$ for the single-hop policy.

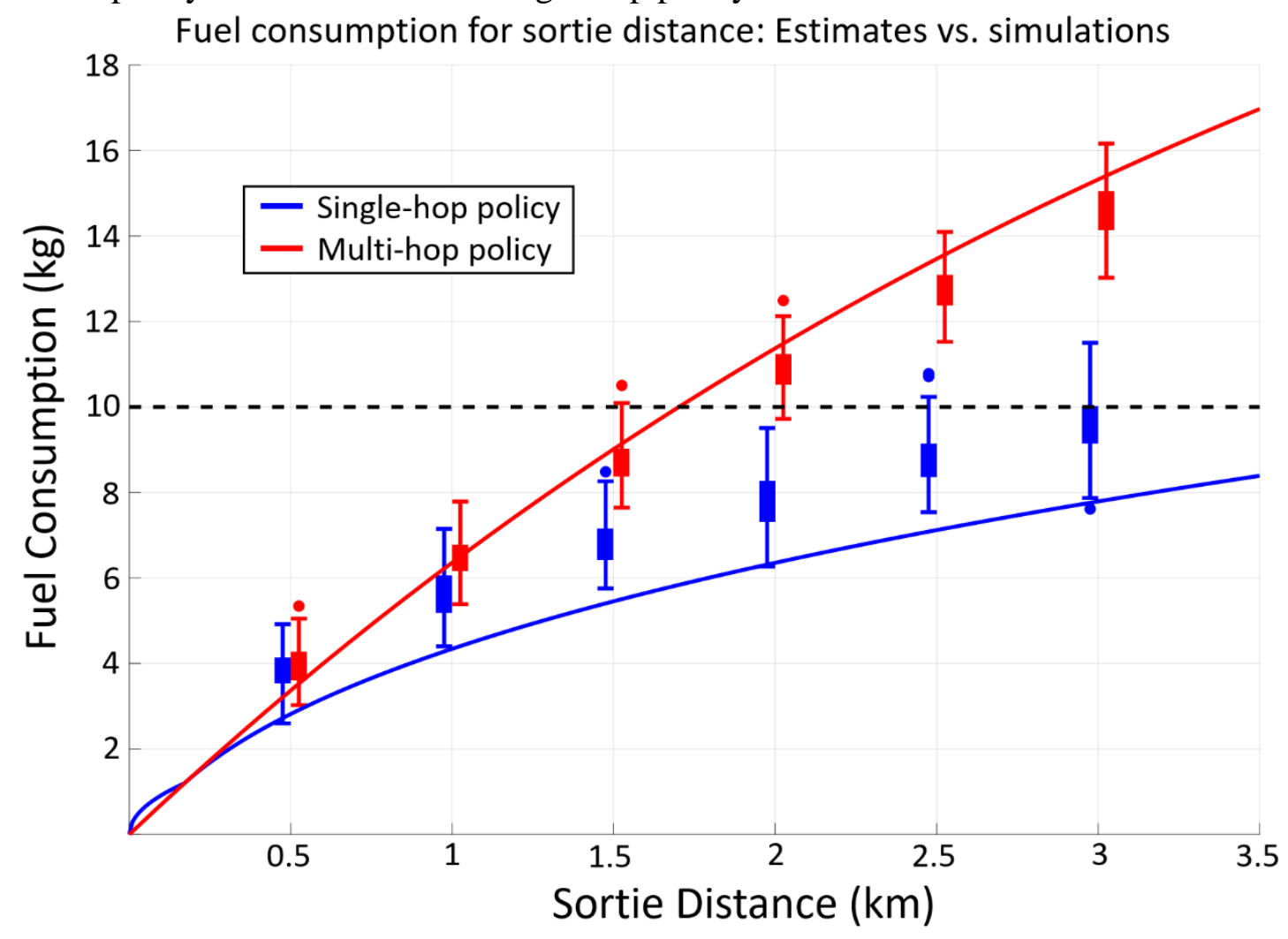

Figure 34: Fuel consumption vs. sortie distance for 1000 Monte Carlo simulations at various sortie distances (box plots), compared to first-order estimates (solid lines).

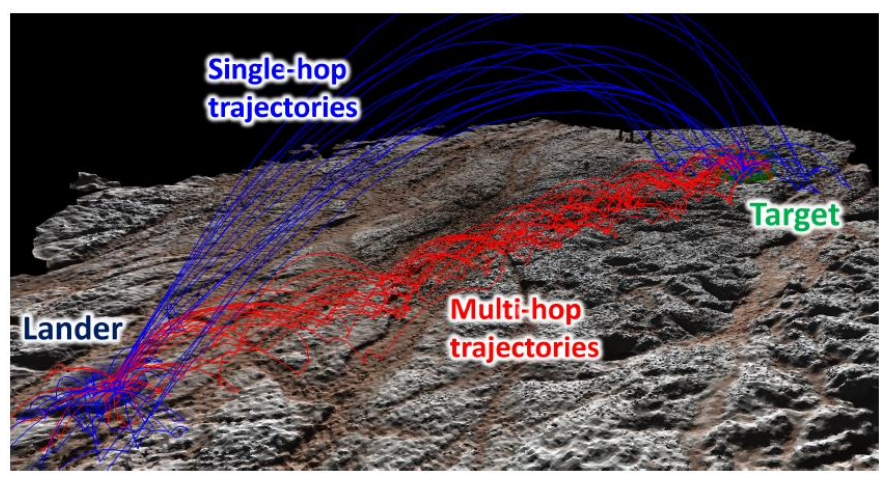

Figure 35: A sampling of Monte Carlo trajectories for each control policy.

\subsection{Localization}

It was noted in Phase I that the challenge of localizing SPARROW on the surface of Europa is non-trivial. The propagation of an IMU alone is unlikely to yield sufficient accuracy due to drift accumulated during the initial thrust, retro-thrust, and tumbling phases. Star or sun trackers may be used in concert with an IMU to refine orientation, but would not yield positional knowledge. The use of terrain relative navigation (TRN), event cameras, or LIDAR, may result in a map against which SPARROW can be localized. This however, will likely require the addition of several cameras to ensure ground-observability during uncontrolled hops. Localization strategies 
will be further investigated in Phase II and combined with the results of propulsion research to determine if a requirement for in-flight stabilization exists.

Effective operational use of SPARROW requires accurate localization at various stages of an observation campaign. While it is proximal to a serviced by the lander, SPARROW must decide on a reference trajectory that will, in expectation, convey it to the first of possibly several remote regions, depending on the chosen control architecture. Tracking this reference trajectory requires determination of an initial pose relative to the lander and servoing, via lowthrust "jogging", to a required initial attitude cone. Uncertainty in achieving this initial pose is, in reality, convolved with the process noise sources of the thrusting mechanism, including propellant contaminants, flow asymmetries, and unqualified propellant-ground effects. Although the commanded thrust profile can be used to provide a reasonable prior on the initial speed at the start of an arc, data collected during flight - for example, high-speed visual odometry - can be used to reduce uncertainty in estimated linear and angular velocities. Other odometric measurement modalities, including lidar and ground-penetrating radar, were deemed during this study to be less likely to achieve uncertainty reduction under the stringent mass/volume/computation budgets and under the Europan environmental conditions. Star trackers, while amenable to surface pose-estimation, can only lock onto the star field at very slow slew rates. Thus, they are likely to be unsuitable for in-flight attitude estimation. A narrow band around the reference trajectory yields a region of potential "first ground impact," contact with which would trigger a series of bounces if the impact velocity is sufficiently high. We assume here that the single-hop architecture, owing to retrothrusting, would have comparably low pose knowledge drift due to bouncing, and consequently the next immediate localization requirement comes at the retrothrust stage. Scheduling of retrothrusts is dependent on timing, altimetry, and velocity estimation to achieve standoff and landing at desired speeds.

\section{Summary and Next Steps}

This report has discussed the scientific impetus behind the exploration of Europa via a mobile asset. The principal finding is that the SPARROW mission concept remains viable but requires further, in-depth, engineering evaluation. A Phase II proposal has been submitted to ascertain answers to open questions.

Phase I presented a science traceability matrix summarizing the effect of sortie range on SPARROW's ability to enable discovery. Challenges of more traditional modes of locomotion were detailed alongside a justification for the use of propulsive hoppers as terrain-agnostic options. The results of first-order calculations and experiments regarding the energetics and efficacy of five propulsion architectures were provided and showed the hot water thruster option to be among the most promising. Finally a discussion of control and observability strategies that may be employed in the tracking of reference trajectories and correcting discrepancies between desired and observed landing locations was given. EDuS prototypes were discussed alongside their energetics and relative efficiencies as functions of ice composition.

The challenge of nozzle and cage icing in cold environments shall was not address in Phase I and is a subject of investigation in the proposed Phase II work. Water thruster firings of the NASA Optical Communications and Sensor Demonstration Program (OCSDP), both during ground-based testing and on-orbit, exhibit excessive icing due to the rapid cooling of exhaust gases. This phenomenon will be explored as part of this task, as well as the viability of nozzle heating, as was found to be sufficient during the OCSDP program (Rowen, et al. 2018).

The current model of hot-water rocket performance is a 1-D analytical model with many simplifying assumptions included. With the more time and resources available in a Phase II study, a more detailed analytic model could be constructed, especially one that allows for more complex nozzle geometry considerations to be considered. Alternatively, a full 2-D 
axisymmetric or 3-D CFD model of the hot water rocket could be constructed. Finally, a sufficient localization strategy for SPARROW is yet to be determined. This is again a core element of the Phase II proposal, requiring both further analytical and experimental approaches.

\section{References}

Ashkenazy, Y. 2016. "The surface temperature of Europa." Physica A.

Buratti, B., and M. Golombeck. 1988. "Geologic implications of spectrophotometric measurements of Europa." Icarus Vol. 75 437-449.

Carlson, R., W. Calvin, J. Dalton, G. Hansen, R. Hudson, R. Johnson, R. McCord, and J. Moore. 2009. Europa - Europa's surface composition. Tucson: University of Arizona Press.

Collins, G., and F. Nimmo. 2009. "Chaos terrain on Europa." In Europa, by R. Papallardo. Tucson: University of Arizona Press.

Doggett, T. 2007. "Global geologic map of Europa." 38th Lunar and Planetary Science Conference. Texas.

Dudzinski, L. 2009. Thermal systems - General Purpose Heat Source. Online, NASA .

Floreano, D., and R. Wood. 2015. "Science, Technology and the Future of Small Autonomous." Nature Vol. 521 460-466.

Hand, K. 2016. Europa Lander study 2016 report. Pasadena: NASA JPL.

Hansen, G. et al,. 2005. "Ice grain size distribution: Differences between Jovian and Saturnian icy satellites from Galilieo and Cassini Measurements." Bulletin of the American Astronomical Sciety Vol 37729.

Heiken, G., D. Vaniman, and B. French. 1991. The Lunar Sourcebook. Cambridge: The University of Cambridge Press.

Hobley, D., J. Moore, A. Howard, and O. Umurhan. 2018. "Formation of metre-scale bladed roughness on Europa's surface by ablation." Nature-Geoscience Vol 11 901-904.

James, K., M. Bodnar, M. Freedman, L. Osborne, R. Grist, and R. Hoyt. 2017. "Hydros: High performance water-electrolysis propulsion for cubsats and microsats." IEEE Space Conference. IEEE.

Kalantari, A., and M. Spenko. 2014. "Modeling and performance assessment of the HyTAQ, a terrestrial/aerial quadrotor." Transactions on Robotics Vol 30 1278-1275.

Kolditz, M., N. Pilz, H. Adirim, P. Rudloff, M. Gorsch, and M. Kron. 2004. "Overview of current hot water propulsion activities at Berlin University of Technology." 2nd International Conference on Green Propellants for Space Propulsion.

Lee, D. , Y. Cheng, and H. Nayar. 2017. "Indirect and dirst planetary illumination modeling for robotic surface exploration sensing." IEEE Aerospace Conference. Big Sky, MT: IEEE. 
McCord, T., G. Hansen, F. Fanale, R. Carlson, D. Matson, T. Johnson, W. Smythe, et al. 1998. "Salts on Europa's surface detected by Galileo's near infrared mapping spectrometer." Science Vol 280 1242-1245.

Molaro, J., M. Choukroun, R. Hodyss, C. Phillips, E. Phelps, J. Lora, G. Meirion-Griffith, and K. Mitchell. 2019 (In press). "The microstructural evolution of water ice in the solar system through sintering." Journal of Geographical Research - Planets.

Moore, J., G. Black, B. Buratti, B. Phillips, C. Spencer, and R. Sullivan. 2009. "Surface properties, regolith, and landscape degradation." In Europa, by R. Papallardo, 329-352. Tucson: University of Arizona Press.

Morren, E., S. Hay, T Haag, and J. Sovey. 1989. "Performance characterization of an engineering model multipropellant resistojet." Journal of Propupsion Vol. 5.

Morrison, D. 1977. "Radiometry of Satellites and of the Rings of Saturn." In Planetary Satellites, by J. Burns, 269-301. Tucson: University of Arizona Press.

Nordheim, T., K. Hand, and C. Paranicas. 2018. "Preservation of potential biosignatures in the shallow subsurface of Europa." Nature - Astronomy Vol 2 673-679.

Paranicas, C., J. Cooper, and H. Garrett . 2009. "Europa's radiation environment and its effects on the surface." In Europa, by B. Papallardo, 529-544. Tucson: University of Arizona Press.

Pugmire, K., R. Shaw, and G. Enos. 1971. "Applied resistojet technology." Journal of Spacecraft Vol. 8.

Rowen, D., S. Janson, C. Coffman, R. Welle, D. Hinkley, B. Hardy, and J. Gangestadt. 2018. "The NASA optical communications and sensor demonstration program: proximity operations." 32nd annual AIAA Conference on Small Satellites. Logan, UT.

Scharf, C. 2017. The Jupiter Vault. Website, Scientific American.

Squyres, S. 2011. Vision and voyages for planetary science in the decade 2013-2022. Decadal Suvey, National Research Council.

Zacny, K. 2018. "Europa Drum Sampler (EDuS)." In Outer Solar System, by V. Badescu. Springer, Cham. 
13 Appendix A (STM)

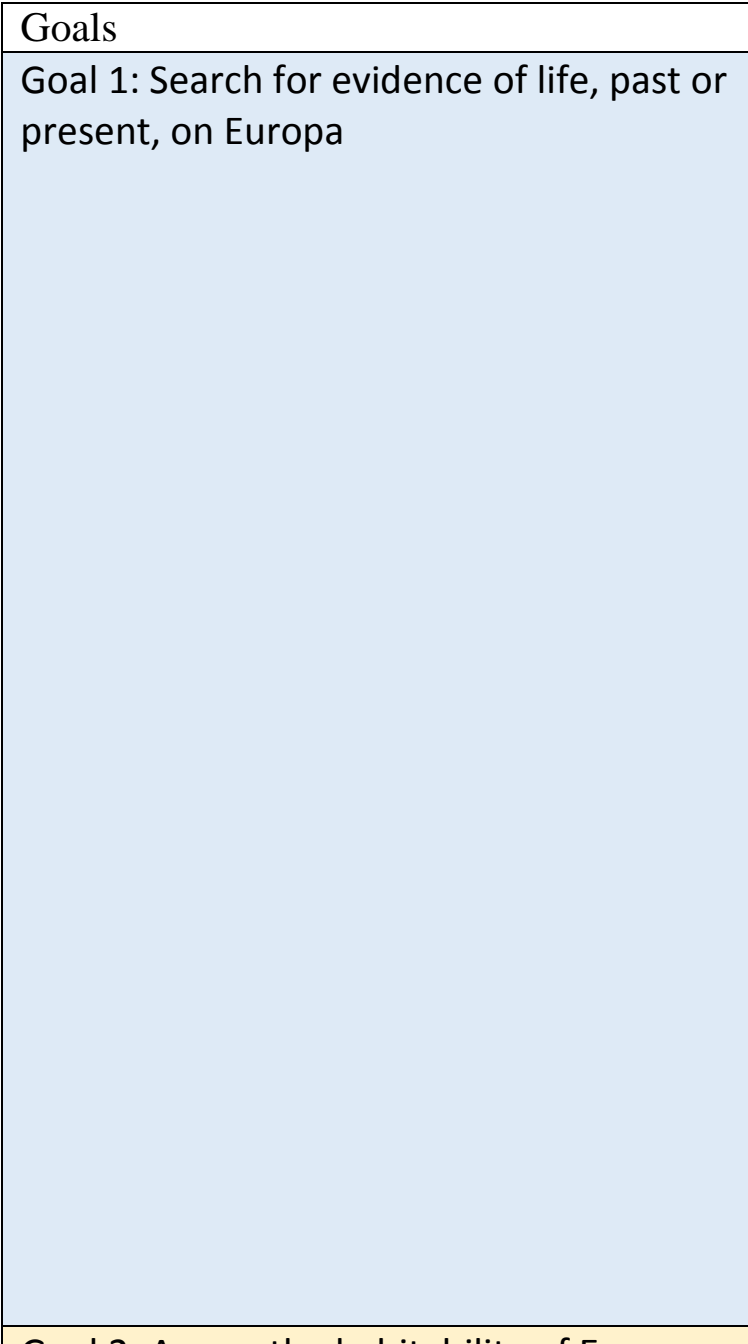

Goal 2: Assess the habitability of Europa
via in situ techniques augmented by surface mobility.

\section{A: Characterize the non-ice composition}

of Europa's near-surface material to determine whether there are indictors of chemical disequilibria and other environmental factors essential for life. 2B: Determine the proximity to liquid water and recently erupted materials at the sampling locations.

2C: Determine habitability across multiple terrain types

\section{Summary}

There is no single test for the presence of life; however, a collection of organic, inorganic, and morphological indictors, taken together could be used to suggest the presence of life. Biosignatures include: patterns among molecules (e.g. carboxylic acids and amino acids) cell-like structures, discoloration, and biominerals such as SiO2. Multiple samples, ideally taken from multiple locations, will be needed to verify results, and spatial variation is likely.

$1 \mathbf{~ k m : ~ G r e a t l y ~ i n c r e a s e s ~ t h e ~ s a m p l i n g ~ a r e a ~ / ~ m e a s u r e m e n t ~ a r e a ~ s u r r o u n d i n g ~ t h e ~ l a n d e r , ~ a n d ~ g r e a t l y ~ d e c r e a s e s ~ t h e ~ r i s k ~ o f ~ b e i n g ~}$ constrained to a less compelling or anomalous workspace. Allows measurements to be taken well beyond any anticipated landerexhaust contaminated region. A $1 \mathrm{~km}$ travel distance enables a partial analysis of one geologic feature or limited interrogation of two units. A partial study would yield a comprehensive understanding of the range of materials contained in a unit, their relative abundance, and potential for preserving biomarkers. Alternatively, two geologic units could be sparsely sampled (e.g. a band and chaos), which likely hold different potential for detecting evidence for life. The ability to reach two units may also alleviate concerns surrounding the choice of which geologic unit holds the most promise for a lander.

$10 \mathrm{~km}$ : A $10 \mathrm{~km}$ travel distance would enable the partial study of two units, or a full interrogation of one unit. This significantly reduces the likelihood of being constrained to a less compelling region or one that is not conducive to preserving biosignatures. A long-distance capability would ease the landing site selection process and allow for the disambiguation of multiple hypotheses surrounding which features hold the most potential for biosignatures. At this distance, multiple unit contacts would be encountered, improving our understanding of the stratigraphic relationships among units. Unit thicknesses, ages, and chemical concentrations may all be interrogated to determine likely material provenance.

$100 \mathrm{~km}$ : A $100 \mathrm{~km}$ travel distance allows the further analysis of two geologic units or a limited sampling of three geologic units. At this distance, observations can be made of very different terrain types, and biosignature analysis can also dramatically increase.

Depending on landing site, a $100 \mathrm{~km}$ distance may also allow regions with very different geologic histories or surface modification histories to be studied.

All: The probability of identifying organic species and biomarkers increases proportionally with traverse range, as does the probability of replicating results. Should multiple organic indicators be found at different locations, comparisons among these indicators would enable the evaluation of diversity of life on Europa, past or present.

Whether or not Europa is actually inhabited, we can also consider whether there are regions that could be habitable for life as we know it. We can look for these by studying the composition of the non-ice material, looking at the proximity to liquid water and recently-erupted materials, and studying multiple terrain types.

$1 \mathrm{~km}$ : As above, a $1 \mathrm{~km}$ travel distance enables a partial analysis of one geologic feature or limited interrogation of two units. A partial study would yield a comprehensive understanding of the compositional range of materials contained in a unit, and their relative abundance. This distance may also allow accessing of nearby plume deposits or other recently-formed geologic features formed through proximity to liquid water. Alternatively, two geologic units could be sparsely sampled (e.g. a band and chaos), which likely hold different compositions. The ability to reach two units may also alleviate concerns surrounding the choice of which geologic unit holds the most promise for a lander.

$10 \mathrm{~km}$ : As above, a $10 \mathrm{~km}$ travel distance would enable the partial study of the composition of two units, or a full interrogation of various locations within one unit. At this distance, multiple unit contacts would be encountered, improving our understanding of the stratigraphic relationships among units. Unit thicknesses, ages, and chemical concentrations may all be interrogated to determine likely material provenance. A $10 \mathrm{~km}$ distance somewhat increases the chance of accessing plume material or recently formed material.

$100 \mathrm{~km}$ : A $100 \mathrm{~km}$ travel distance allows the further analysis of two geologic units or a limited sampling of three geologic units. At this 


\begin{tabular}{|l|l|}
\hline & \\
\hline $\begin{array}{l}\text { Goal 3: Characterize surface } \\
\text { morphological and geophysical properties }\end{array}$ & $\begin{array}{l}\text { 3A: Observe the properties of surface } \\
\text { materials and sub-meter landing and } \\
\text { mobility hazards within the vehicle's } \\
\text { proximity. Connect local properties with } \\
\text { those seen from flyby remote sensing. } \\
\text { 3B: Characterize dynamic process of } \\
\text { Europa's surface and ice shell over the } \\
\text { mission duration within different terrains } \\
\text { to understand exogenous and } \\
\text { endogenous effects on the } \\
\text { physiochemical }\end{array}$ \\
\hline
\end{tabular}

distance, observations can be made of very different terrain types, and compositional analysis can also dramatically increase. Depending on landing site, a $100 \mathrm{~km}$ distance may also allow regions with very different geologic histories or surface modification histories to be studied, and largely increases the possibility of accessing nearby plume material.

All: Identifying multiple components of surface composition and accessing plume material increases proportionally with travel range, as does the probability of replicating results.

Studying the physical properties of Europa's surface and the dynamic processes that modify it helps us understand the context for any potential biosignatures. In addition to surface measurements, the subsurface can be probed for proximity to liquid water with a sounding package.

$1 \mathrm{~km}$ : As above, a $1 \mathrm{~km}$ travel distance enables a partial analysis of one geologic feature or limited interrogation of two units. A partia analysis will allow different physical environments to be analyzed. Alternatively, two geologic units could be sparsely sampled (e.g. a band and chaos), which likely hold different physical structures. This distance will allow small variations in dynamic processes and in distance for subsurface measurements by a seismic package.

$10 \mathrm{~km}$ : As above, a $10 \mathrm{~km}$ travel distance would enable the partial study of the composition of two units, or a full interrogation of various locations within one unit. At this distance, multiple unit contacts would be encountered, improving our understanding of the physical properties of the surface and beginning a survey of dynamic process variation. A $10 \mathrm{~km}$ distance inceases the utility of subsurface measurements. 